\title{
EFSUMB Guidelines and Recommendations on the Clinical Use of Ultrasound Elastography. Part 1: Basic Principles and Technology
}

Authors

Affiliations
J. Bamber ${ }^{1}$, D. Cosgrove ${ }^{2}$, C. F. Dietrich ${ }^{3}$, J. Fromageau ${ }^{1}$, J. Bojunga ${ }^{4}$, F. Calliada ${ }^{5}$, V. Cantisani ${ }^{6}$, J.-M. Correas ${ }^{7}$, M. D’Onofrio ${ }^{\text {, }}$, E. E. Drakonaki ${ }^{9}$, M. Fink ${ }^{10}$, M. Friedrich-Rust ${ }^{11}$, O. H. Gilja ${ }^{12}$, R. F. Havre ${ }^{13}$, C. Jenssen ${ }^{14}$, A. S. Klauser ${ }^{15}$, R. Ohlinger ${ }^{16}$, A. Saftoiu ${ }^{17}$, F. Schaefer ${ }^{18}$, I. Sporea ${ }^{19}$, F. Piscaglia ${ }^{20}$

Affiliation addresses are listed at the end of the article.

Key words
elastography
elasticity
radiation force
shear wave
stiffness
shear modulus
strain
transient
ultrasonography

Bibliography

DOI http://dx.doi.org/ 10.1055/s-0033-1335205

Published online: 2013

Ultraschall in Med 2013; 34 : 169-184 @ Georg Thieme Verlag KG Stuttgart · New York ISSN 0172-4614

\section{Correspondence}

\section{Dr. Jeffrey Bamber}

Ultrasound and Optics Team, Division of Radiotherapy and Imaging, Institute of Cancer Research and Royal Marsden NHS Foundation Trust Downs Road, Sutton, Surrey SM2 5PT

United Kingdom

Tel.: +44/20/86613343(or$3704)$

jeff@icr.ac.uk

\section{Zusammenfassung \\ $\nabla$}

Der technische Teil dieser Leitlinien und Empfehlungen, der unter der Federführung der EFSUMB entstanden ist, enthält eine Einleitung zu allen physikalischen Grundlagen und Methoden der aktuell kommerziell erhältlichen Ultraschall-Elastografie-Technologien. Unterschiede in der Schersteifigkeit sind der wesentliche physikalische Mechanismus, dem der Gewebekontrast in allen Elastogrammen zu Grunde liegt. Die Beziehung zu alternativen Technologien wird und die Vorteile der angewandten Methode herausgearbeitet. Die praktischen Vorteile und Nachteile jeder Technik werden beschrieben und Empfehlungen zur Optimierung der Scantechnik, der Bildqualität und der Bildinterpretation gegeben, ebenso werden einige der bekannten Artefakte dargestellt.

\section{Introduction}

Palpation is an ancient diagnostic technique. It is mentioned in the Edwin Smith Surgical Papyrus dating from the seventeenth century B.C. [1], and Hippocrates was reported to use it for battle injuries, identifying that if the bone is not visible then palpation can assist in locating the weapon mark and, for head injuries, determine whether the cranium beneath the flesh is strong or weak. Palpation is of course still valuable and is essentially used in the same way that it has been for thousands of years, both clinically and in self-examination. Tactile feedback is also a seamless, routine and essential component of effective surgery. However, its application is limited to accessible organs, it provides limited disease detectability and spatial discrimination, and the interpretation of the information is subjective.

Elastography, the science of creating noninvasive images of mechanical characteristics of tissues, aims to overcome these limitations and has been

\section{Abstract \\ $\nabla$}

The technical part of these Guidelines and Recommendations, produced under the auspices of EFSUMB, provides an introduction to the physical principles and technology on which all forms of current commercially available ultrasound elastography are based. A difference in shear modulus is the common underlying physical mechanism that provides tissue contrast in all elastograms. The relationship between the alternative technologies is considered in terms of the method used to take advantage of this. The practical advantages and disadvantages associated with each of the techniques are described, and guidance is provided on optimisation of scanning technique, image display, image interpretation and some of the known image artefacts. rapidly evolving in recent years. Therefore, in 2011 the European Federation for Ultrasound in Medicine and Biology (EFSUMB) felt the need to develop a set of recommendations for the use of elastography, given the growing general interest, number of available techniques and level of scientific evidence in this field, following a process similar to that utilized for Contrast Enhanced Ultrasound $[2,3]$. The steering committee formed in 2011 selected the topics to be included and invited a team of experts with significant publication records and skill in the field, from throughout Europe. The manuscript, which was prepared by e-mail and telephone communication, was discussed in a consensus meeting held in Bologna in September 2012.

The project was partially supported by the ultrasound industry, as mentioned in the acknowledgements. The industry, however, had no influence in deciding the content of the manuscript, which is solely under the responsibility of the authors. 
The current set of guidelines is divided into two parts. Part 1, which follows below, introduces the technical background and principles. This is important since various different techniques exist which may all be described as elastography, and yet each operates in a different way and might therefore have different applications. Part 2 [4] reports the main clinical applications of the various elastographic methods as of 2013 and is published in the next issue of this journal. EFSUMB hopes these recommendations will help users and would-be users of elastography to understand the basic principles and limitations of the method, and to apply it and successfully interpret the findings across the most commonly used clinical applications.

Despite the plethora of different elastography methods that have become available they all aim to display contrast for, or measure quantities related to, the shear elastic modulus of tissue (described on p. 171 and in the online appendix). In this sense, all of the methods are unified, justifying the use of the common term, elastography. A second unifying feature is in the signal processing used; for all of the methods in common use the signal processing to create an elasticity measurement or image begins with an ultrasound measurement of time-varying tissue displacement. The methods are then classified according to what they do with this measurement, either displaying it directly as the imaged quantity, using it to calculate and display strain, or using it to calculate and display an image of shear-wave speed. The last of these is a quantitative image and is the only one that requires the creation of a shear-wave, which in turn requires the use of a dynamic force. The others may use a dynamic force but can also work with a static, or nearly static, force. All of these terms will be explained in this article.

\section{Basic principles of elastography \\ $\nabla$}

The mechanical properties sensed by palpation and elastography are associated with the elastic restoring forces in the tissue that act against a type of deformation (shape change) known as shear. Two types of shear may be defined. Referring to $\bullet$ Fig. 1: in a "simple shear" results from a simple shearing force (arrow) such as that applied to displace a single location on or within the body - an object of square cross-section (dashed outline) is deformed to one that has a trapezoid cross-section (solid outline) without a change in its volume. b depicts "pure shear", resulting from a compressive force (arrows) such as that applied to displace the whole surface of the body - the same object as in a will be compressed axially (vertically), resulting in a lateral (horizontal) expansion that conserves volume (solid outline). In the case of pure shear, the shearing nature of the deformation is less obvious than in the case of simple shear. It can be seen, however, by observing the deformation of an element of the medium that has a different shape to the rectangular grid normally used to illustrate the lateral expansion that is created when an incompressible medium is compressed axially. The inner diamond (dotted outline) in the undeformed case in $\mathbf{b}$ is a shape that is useful for visually revealing pure shear; the shear deformation becomes apparent if one rotates the deformed element (the flattened diamond drawn with the solid outline) so that its bottom-left boundary is parallel to that of the undeformed element, and if one then rotates the whole diagram clockwise by 45 degrees, as shown in c. The actual tissue deformations generated in elastography are more complex than these simple idealisations, although those produced by acoustic radiation force most closely approximate simple shear and those produced by using the ultrasound transducer to displace the tissue surface most closely approximate pure shear.

No method directly images the shear elastic properties of tissue; elastography methods use an existing modality, such as ultrasound, to observe the internal tissue deformations that occur in response to an applied force, and convert the resulting information to a suitable form for display. A wide variety of approaches has evolved, both for applying the force and for measuring and displaying the tissue's response.

Elastography uses ultrasonic imaging to observe tissue shear deformation under conditions of one or both of the types of shear illustrated in 0 Fig. 1, currently in a real-time two-dimensional image sequence, after applying a force that is either dynamic (e. g. by thumping or vibrating) or varying so slowly that it is considered "quasi-static" (e.g. by probe palpation). The deformation may be represented in an elasticity image (elastogram), or as a local measurement, in one of three ways:

1. tissue displacement may be detected and displayed directly, as in the method known as acoustic radiation force impulse (ARFI) imaging,

2. tissue strain (defined on p.174) may be calculated and displayed, producing what is termed strain elastography (SE), or

3. in the dynamic case only, the data may be used to record the propagation of shear-waves, which are used to calculate either a) regional values of their speed (without making images) using methods referred to herein as transient elastography (TE) and point shear-wave elastography (pSWE), or

b) images of their speed using methods referred to herein as shear-wave elastography (SWE) which includes $2 \mathrm{D}$ SWE and $3 \mathrm{D}$ SWE.

These shear-wave speed methods could all be grouped under the term SWE. However, the literature uses SWE to describe methods that make images of shear-wave speed using excitation by acoustic radiation force, which is a convention broadly used in this paper. For the same reason, the term pSWE has been used for the (non-imaging) regional measurement of shear-wave speed using acoustic radiation force excitation, even though the term ARFI quantification has been used in the literature to describe this method.

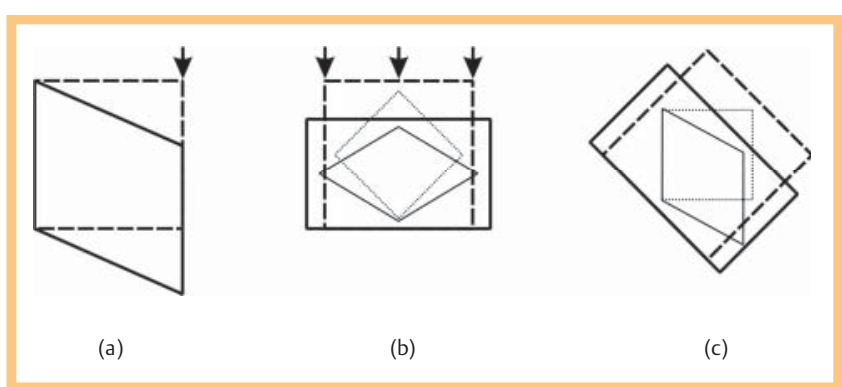

Fig. 1 Idealisations of two types of shear deformation: a "simple shear" resulting from a simple shearing force (arrow) and b "pure shear" resulting from a compressive force (arrows) applied to displace the whole surface of the body. The shear deformation present in case $\mathbf{b}$ is revealed in $\mathbf{c}$ by rotating the deformed element (the flattened diamond drawn with the solid outline) so that its bottom-left boundary is parallel to that of the undeformed element, and then rotating the whole diagram clockwise by 45 degrees (see the text for a more detailed description). (Inspired by Steven Dutch's explanation of shear strain, at https://www.uwgb.edu/dutchs/ structge/shear.htm.) 


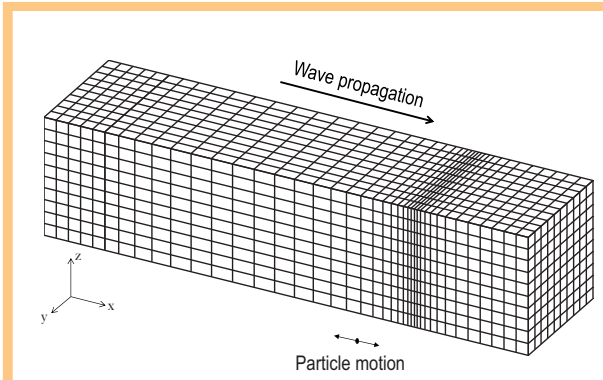

(a)

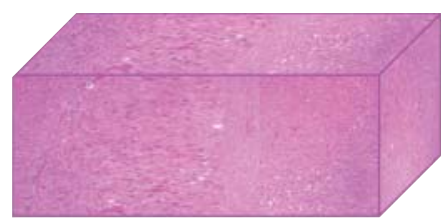

(b)
Fig. 2 a A longitudinal wave travelling from left to right moves as a transference of pressure and density variations from one tissue element to another, seen here as a bunching of compressed tissue elements, where the pressure is at a maximum, and a dilation of expanded tissue elements just prior to (to the left of) these, where the pressure is at a minimum. As the wave passes a given point, the individual elements (or "particles") of tissue oscillate along the direction of propagation. $\mathbf{b}$ The compression and dilation shown in a has been applied to a simulated block of liver tissue formed from stained histological sections.

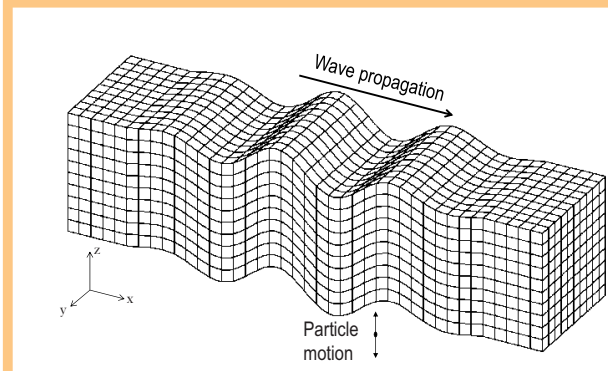

(a)

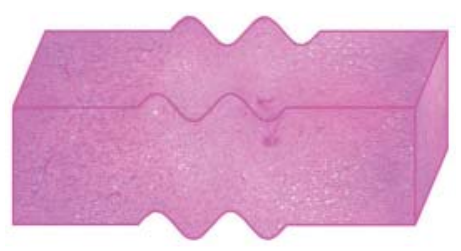

(b)
Fig. 3 a A simple shear wave travelling from left to right moves as transference of variations in shear force and deformation from one tissue element to another, seen here as a distortion from the cubic shape of the tissue elements. As the wave passes a given point, the individual elements (or "particles") oscillate across the direction of wave propagation. b The deformation shown in a has been applied to a simulated block of liver tissue formed from stained histological sections.
ARFI, SE, TE, pSWE and SWE are explained in detail below. Elastograms may be displayed separately from B-mode scans, or the two superimposed, using colour to distinguish them.

\section{Shear propagation in soft tissues}

In this section the relationship between ultrasonic imaging and elastography is explained.

In ultrasonic imaging [5-11] a varying pressure at the tissue surface causes a change in density, and both perturbations travel together as a longitudinal wave ( $\bullet$ Fig. 2 ). Its speed $c_{l}$ (about $1540 \mathrm{~ms}^{-1}$ ), is determined almost entirely by the tissue's density $\rho$ and the bulk modulus of elasticity $K$ (see the online appendix for equations). The image shows the strengths of echoes, which are produced by local variations in $\rho$ or $K$, as a function of tissue depth estimated from the time of the echo and the value of $c_{l}$. In elastography a directional force is applied to the tissue (arrows in $\bullet$ Fig. 1), which causes shear deformation that propagates as a shear-wave. An example corresponding to simple shear is shown in $\bullet$ Fig. 3 (shear propagation in reality can be much more complex than this, as described on p. 182 and in the online appendix). In an ideal situation (an elastically homogeneous medium of infinite spatial extent) the speed of propagation $c_{s}$ of the (transverse) shear-wave is determined by $\rho$ and the shear elastic modulus $G$. Because in soft tissues $G$ is much smaller than $K$, shearwaves travel about 1000 times slower than longitudinal waves, typically in the range $1-10 \mathrm{~ms}^{1}$ (see appendix). Unlike longitudinal waves, at ultrasonic frequencies shear-waves are attenuated rapidly in soft tissue. Hence very low frequencies, often below $1 \mathrm{kHz}$ (which includes static deformations), must be used for elastography. Importantly, non viscous pure fluids do not propagate shear-waves.

When an ultrasound transducer is used to displace the tissue surface, this is referred to as a quasi-static compression. It generates a complex pattern of shear deformation, consisting of both pure and simple shear ( $\bullet$ Fig. 1 ), that propagates at speed $c_{s}$, and can be regarded as equivalent to a static compression so long as the palpation rate is slow relative to the propagation time to the depth of interest.

Why can elastograms be made from ultrasound images? It is a fortunate coincidence of nature that the above key differences in the propagation properties of longitudinal and shearwaves combine helpfully to make ultrasound elastography possible:

- Speckle, ubiquitous in soft tissue ultrasound images, arises from diffusely distributed variations in $K$ and $\rho$, and provides image markers that follow the shear deformations.

- The relatively low attenuation of ultrasound allows high-frequency (and hence high resolution) ultrasound imaging, which can detect tiny shear-wave displacements and resolve differences in the motion of neighbouring tissue elements.

- The relatively high speed of ultrasound allows echo data to be acquired rapidly enough to observe the tissue displacements at clearly defined phases of the shear deformation.

\section{Tissue shear properties for elasticity imaging}

Measurements of the shear modulus $G$, and its close relative Young's modulus $E$ (see online appendix), may be made noninvasively if one can monitor the displacements of the tissue-elements as a function of time as a shear-wave passes multiple points along its path. For example, in TE and SWE, $G$ is obtained 
from shear-wave speed measured as the difference in shearwave arrival time at two or more points separated by known distances.

In addition, both the percentage deformation (known as strain) and the displacement generated are directly dependent on the value of $G$ for a given force, and both have been employed, in different elastography techniques, to display tissue shear modulus contrast. A common technique is to apply a uniaxial force to the tissue, and to use ultrasound to measure and map the resulting displacement. In some techniques displacement is displayed directly as an image. As explained on p.179, this is convenient when the displacement is highly localised, such as in ARFI displacement imaging, but where the whole top surface of the body is displaced it is better to convert the internal tissue displacements to a strain image since, for this situation in a homogeneous medium, strain will be constant whereas displacement decays with depth.

Although a dynamic force is mandatory for generating a shearwave and thus measuring or imaging its speed (as in SWE), when imaging displacement or strain the force may be dynamic, quasi-static or static. Usually the force is not known, and neither are the positions of, or friction at, the tissue boundaries. As a result, techniques that display images of displacement or strain do not produce values for $G$. Nevertheless, they generate image contrast that is directly related to $G$, albeit with influences of other factors that create artefacts that need to be understood. The SWE images also contain artefacts, as described on p. 181.

Biological tissues are much more complicated than the above description which assumes a linearly elastic, homogeneous, isotropic, infinite, continuous medium. The practical situation departs from these assumptions in that:

a) the force-deformation relationship is usually both nonlinear and time dependent (the latter due to the phenomena of viscoelasticity and poroelasticity, described in the online appendix),

b) elasticity, in fact, varies spatially and with direction (a phenomenon known as anisotropy - see the online appendix),

c) tissue boundaries and structure change the relationship between shear-wave speed and $G$, due to the phenomena of guided shear-wave propagation and shear-wave scattering (see the online appendix), and

d) tissues may be rendered mechanically discontinuous by anatomical features such as fluid collections, organ or tumour boundaries, and scars.

All these can produce artefacts in the current generation of elastography techniques. Current research aims to take account of them, to gain additional information and to afford elastography the ability to image new tissue characteristics, providing considerable potential for future evolution of elastography.

\section{What makes elastograms different from ultrasound images?}

While the bulk modulus $K$ varies by little more than $12 \%$ over the whole range of soft tissues, the shear modulus $G$ varies by a factor of more than $10^{5}$. This provides the potential for images with enormous tissue contrast, if they can be made with relatively good signal to noise ratio [12]. Furthermore, the speed and absorption of ultrasound waves in soft tissue are largely determined at the molecular fluid level. Shear stress is not sustained by fluids, and is transmitted by the connected structural matrix within tissues (extracellular matrix, microvasculature, membranes, etc.). The integrity of this matrix, and the tension placed upon it by micro- vascular and interstitial pressure, are important for shear-wave propagation, but not for the generation of ultrasound echoes.

\section{Technology of ultrasound elastography}

The various current commercially available ultrasound elastographic methods ( $\bullet$ Table 1 ) may be complementary, particularly in relation to the shear properties of tissues that each is most suited to evaluate. They possess different physical performance properties, artefacts, limitations and suitability for specific clinical applications.

\section{Tissue displacement estimation}

$\nabla$

All current commercial elastography systems need to measure tissue displacement. The various systems differ in how the displacement is used; it may be imaged directly, converted to strain, or used to detect the time of arrival of shear-waves and hence their speed. The most common methods in use for elastography, RF echo correlation-based tracking and Doppler processing, are described in 0 Fig. 4, but tracking of the echo envelope and the echo-envelope fluctuation rate can also be used [13]. An axial displacement image is formed by generating a displacement-versus-depth line for all RF A-lines in a two-dimensional (2 D) echo image sequence, where each line is obtained by repeating the process explained in $\bullet$ Fig. 4 for multiple (usually overlapping) reference windows or Doppler gates at different depths. Echocorrelation tracking may also be implemented in the lateral di-

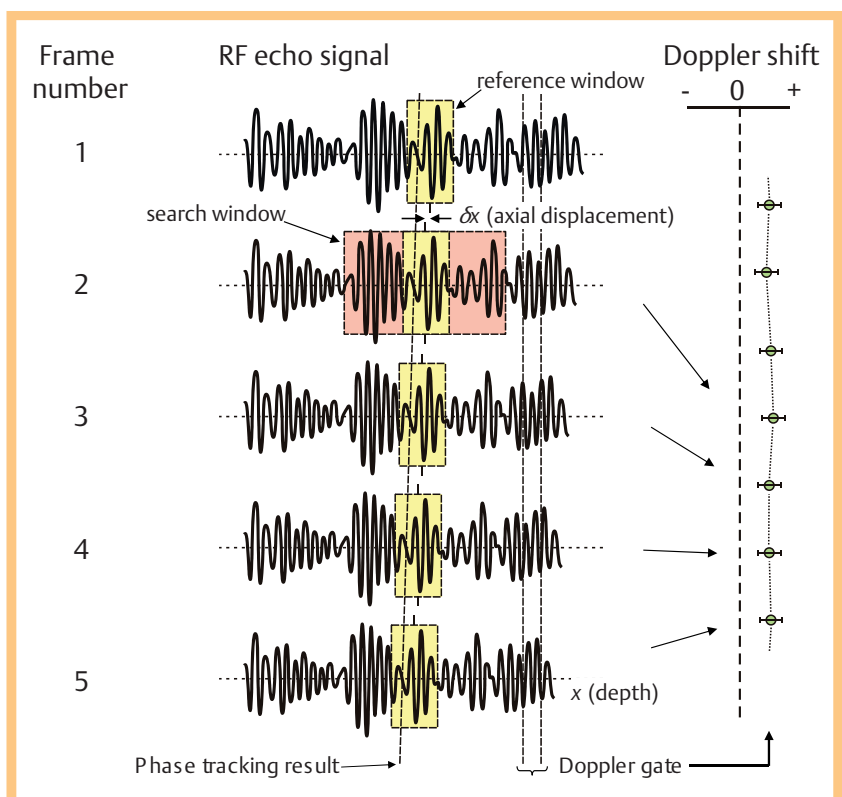

Fig. 4 The two main methods for estimating displacement used in elastography are RF-echo correlation tracking and Doppler processing. In this illustration, stress is applied to the tissue such that an axially compressive strain, that is constant with depth, is successively generated in equal amounts from one RF-echo image to the next; the echo signals shown represent the evolution of the same RF-echo line over 5 images. Doppler processing uses the correlation between a gated region of the RF-echo signal and the same region in the same echo line at a previous time to measure displacement. RF echo-tracking explores a search window (pink) to locate the position of the echo pattern that best correlates with that in a reference window (yellow) within the echo line at a previous time; the shift required to maximise this correlation is the required axial displacement, $\delta x$. 
Table 1 The types of elastography described in this paper.

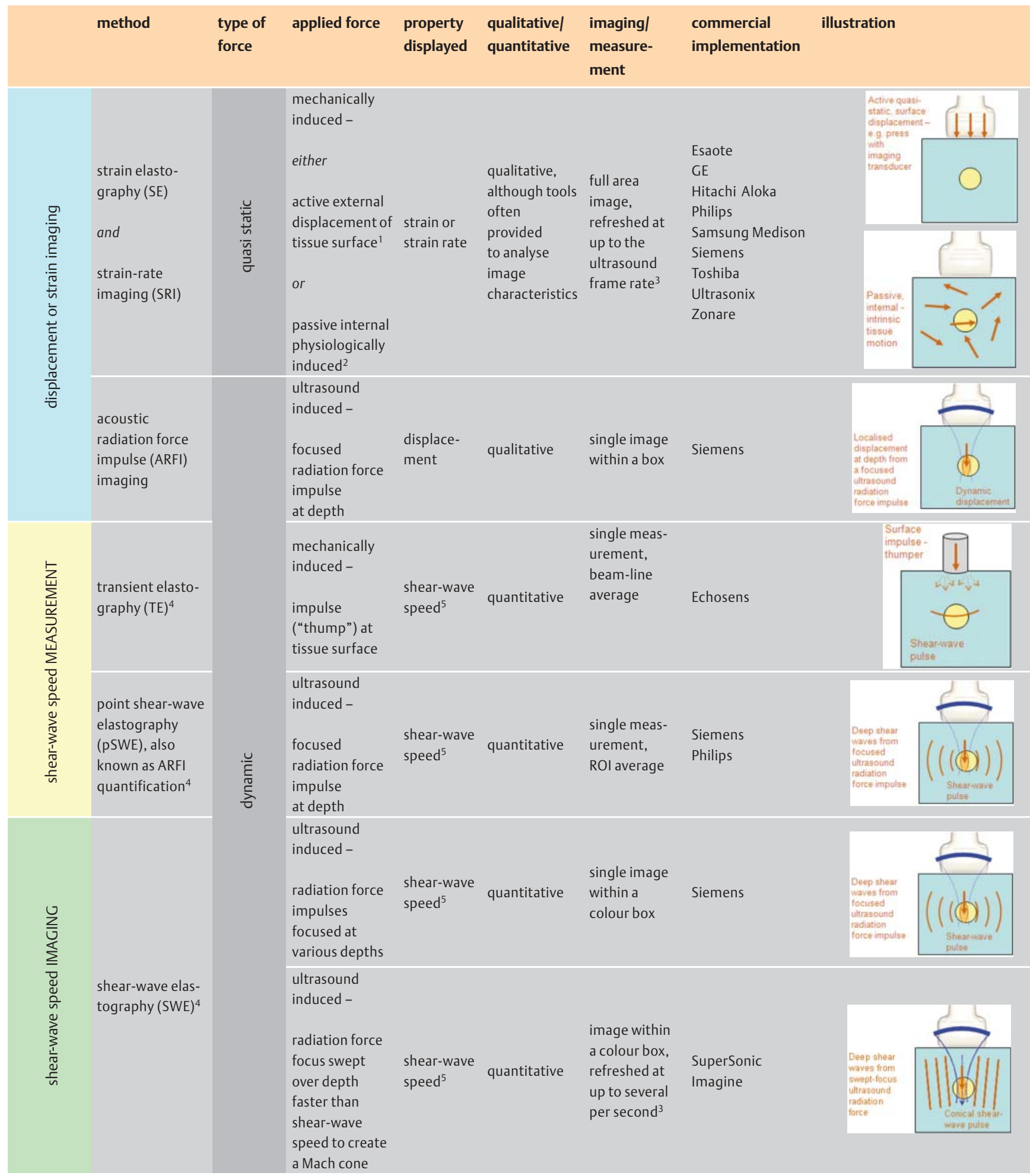

1 Palpation, balloon expansion etc., whether body surface or intracavity.

${ }^{2}$ Cardiovascular or respiratory pulsation, or muscular contraction.

${ }^{3}$ The term "real time" has been avoided here as, unlike real-time ultrasonography (and as explained in the text), the elastography systems described in this paper are not yet suitable for rapid searching through large volumes nor for observing rapid tissue motion within a sequence of elastograms.

${ }^{4}$ The term shear-wave elastography (SWE) has been used here according to the current literature, where it refers to methods that make images of shear-wave speed using radiation force excitation. Thus it includes 2D SWE and 3 D SWE. The term point shear-wave elastography (pSWE) has been used for the method where a regional average only (no image) of shear-wave speed is measured using radiation force excitation. This emphasises that it is essentially a SWE method, even though in the literature it has been referred to as ARFI quantification. Transient elastography (TE) also measures shear-wave speed without creating an image but, since it uses a surface mechanical force rather than acoustic radiation force, it has not been classified here under the term SWE.

${ }^{5}$ Shear-wave speed may be converted to shear modulus or Young's modulus under assumptions explained in the text and the online appendix. 

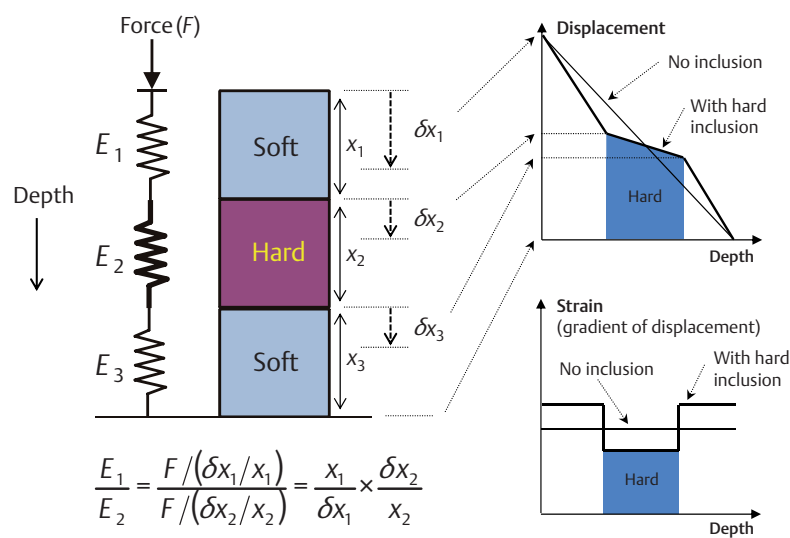

(a)

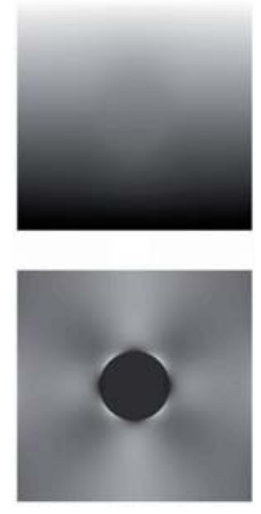

(b)
Fig. 5 a The relationship between depth-dependent displacement and strain, based on a simple spring model and an (over-simplified) Hooke's law assumption that applied force $(F)$ is identical for both soft and hard springs at different depths and that the strain experienced by each spring is inversely proportional to its elastic constant $E$. Displacement imaging requires visual comparison of gradients, which is difficult in a grey scale or colour image, whereas strain imaging easily separates the hard and soft regions. b Grey-scale displacement (upper) and strain (lower) images of a simulated hard tumour of circular crosssection in a homogeneous soft background, where bright represents large displacement and high strain respectively. Note that the inhomogeneous background in the strain image is due to stress concentration, an artefact characteristic of strain imaging that is described in the text. (Images in $\mathbf{b}$ courtesy of L. Garcia). rection, and be extended to $2 \mathrm{D}$ reference and search windows (or even $3 \mathrm{D}$, for data available from $4 \mathrm{D}$ probes). The lateral displacements that this is designed to follow are measured with much poorer precision than the axial displacements, due to the lack of availability of echo phase information. Nevertheless, such tracking is helpful for noise reduction in axial displacement measurement, as described on p. 174.

When stress is applied to tissue, whether static or dynamic, strains occurs and the echoes do not remain correlated, which creates displacement noise - the greater the strain, the greater this decorrelation-induced displacement noise. Under the condition that tissue is nearly incompressible, axial compression results in lateral expansion and thus lateral displacement, which adds to this noise. Substantial decorrelation noise also occurs in freehand tissue compression (used in SE, described on p.174) if the probe motion is not solely axial, for example, if it rotates or moves across the tissue either laterally or in elevation. As a result, estimating displacement from $1 \mathrm{D}$ (axial) tracking with minimal noise requires careful probe motion in a uniaxial direction only, and careful choice of palpation rate, RF-frame acquisition rate, and the sizes of the reference and search windows. In current elastography systems $2 \mathrm{D}$ tracking is used to improve the precision of axial displacement estimates by reducing decorrelation noise due to lateral expansion and probe motion; $3 \mathrm{D}$ tracking is needed to do the same in the elevation direction. Each manufacturer also tends to implement its own preferred method for displacement image optimization.

\section{Quasi-static methods \\ $\nabla$}

\section{Strain elastography (SE): quasi-static strain imaging}

In quasi-static strain imaging, axial and lateral tracking is typically applied between each pair of RF-echo frames, as described above, and the lateral displacements are discarded leaving a sequence of axial displacement images. Each axial displacement image is then converted to a strain image by passing a movingwindow (the strain estimator window) down each image line, to calculate the local axial gradient of displacement at each window position. The size of the strain-estimating window is chosen as a compromise between strain image resolution (small window) and good strain signal-to-noise ratio $(S N R=$ the ratio of mean

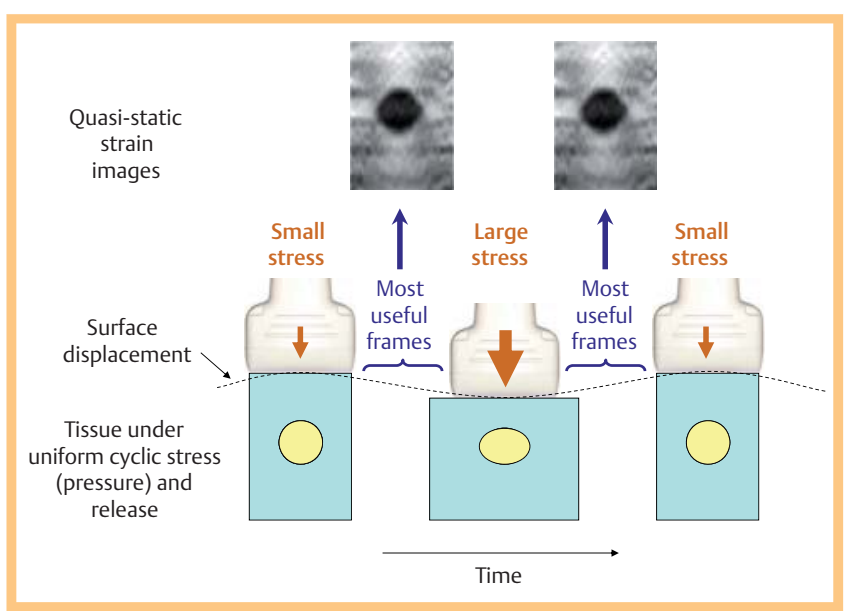

Fig.6 Quasi-static strain elastography using cyclic axial palpation with the transducer. When the tissue is placed under relatively large stress by pressing on it with the transducer, it strains more than when the stress is reduced during the release phase of the cycle. A hard region strains less than surrounding soft material, causing it to appear dark in an image where high strain is displayed as bright. The most useful frames for computing strain images with good signal-to-noise ratio are those with a near constant rate of surface displacement. Note that the inset strain images are of a real phantom containing a stiff cylindrical inclusion, and contain decorrelation noise. Nevertheless, the stress concentrations mentioned in Fig. 5 and in the section on artefacts on p. 175 are clearly visible.

strain to standard deviation of strain for an elastically homogeneous region). In some systems this choice may be made by the operator, in others it is preset by the manufacturer. The relationship between strain and displacement is illustrated in $\bullet$ Fig. 5 . The imaging impact of choosing different window sizes is discussed and illustrated on p. 176 in the section on strain image quality.

The imaging of strain generated by palpation with the ultrasound transducer is illustrated in $\bullet$ Fig. 6. Usually the sign of the strain (i. e. positive for axial expansion, negative for axial contraction) is discarded and only its magnitude is displayed, because this can be easily integrated over time; a high level of persistence (time integration) is generally applied to strain images to improve their SNR. The transducer may alternatively be held stationary to im- 

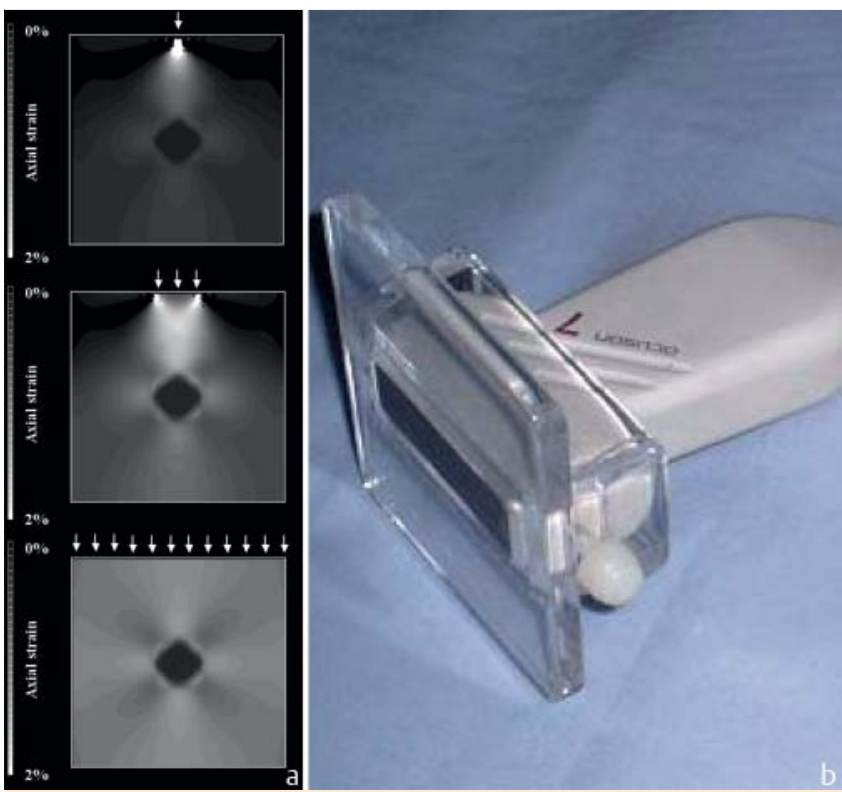

Fig. 7 a A narrow compressor (upper image) generates strain that is concentrated below the compressor and decays rapidly with depth. A compressor of lateral and elevational width equal to or greater than the lateral extent of the region imaged is needed to create the most homogeneous strain distribution (lower image) and to maximise the depth of stress penetration (courtesy of AF Kolen [65]). b A transducer footprint extender [30], which helps to achieve this in practice. If a footprint extender is not available then placing a finger of the hand that is not holding the transducer, on the skin on either side of the transducer, and moving these with the transducer, helps obtain deeper strain penetration.

age strain generated by internal physiological pulsations (e.g. cardiovascular, respiratory) or muscular contractions. However, when this is done, in general the origin and direction of the stress is unknown; therefore only the axial component of displacement and strain are measured. There are also likely to be more sources of decorrelation noise than when a simple uniaxial stress is applied by moving the transducer, although techniques for obtaining good quality strain images under such conditions have been published and are employed in commercial systems.

Although strain images are displayed at frame rates up to the ultrasound frame rate, the need to average over many frames while moving the tissue means that strain imaging is not as suitable as real-time ultrasonography for observing tissue motion or for rapidly exploring a volume. The latter problem may be solved by 3 D elastography [14].

\section{Strain-rate imaging (SRI)}

Strain-rate imaging is essentially identical to quasi-static strain imaging; its name comes from being implemented on cardiology scanners that already possess tissue Doppler capability, by taking the axial gradient of the tissue velocity image [15]. In fact, all real-time strain imaging methods display strain per sample-interval and thus are sensitive to strain rate. The sign of the strain is preserved in strain-rate images, which assists the study of muscular contraction and relaxation. It can distinguish between actively contracting and passively moving tissue, such as the gastrointestinal wall, which currently is the only clinical application $[16,17]$.

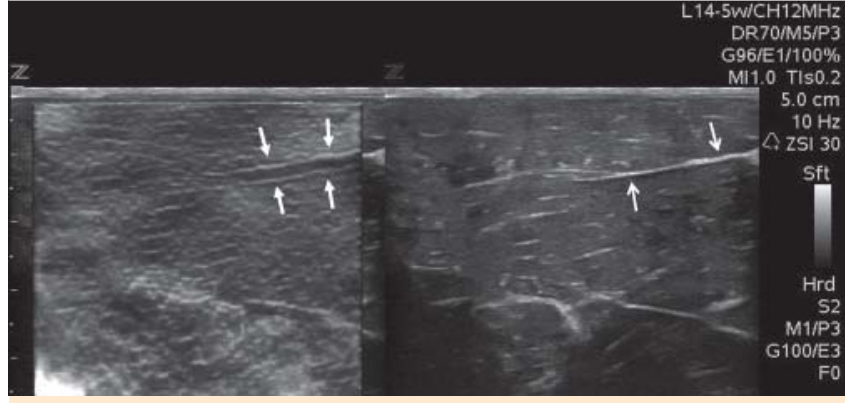

Fig. 8 Stress and strain concentrations (and possibly high strain at slippery boundaries) appear to exist in the strain image of a turkey breast on the left as a bright line (i. e. high strain, suggesting soft tissue) superficial and deep (arrows) to many of the dark bands (i. e. low strain, suggesting hard tissue) that correspond to many of the echogenic (probably stiff and possibly slippery) fibrous septa visible in the B-mode image on the right (arrows). Understanding physical phenomena such as these helps make sense of clinical elastography. See also Fig. 5, 6.

Artefacts - what influences strain, or strain rate, other than tissue elasticity?

Because the stress is not homogeneously distributed within the tissue, strain-image contrast (brightness or colour) is influenced by effects other than tissue elasticity. Friction between the transducer and skin produces less strain close to the surface than when good lubrication is present; a compressor of limited size generates stress and strain with limited penetration and poor homogeneity ( $\bullet$ Fig. 7); and soft tissue strains more when it is adjacent to hard tissue than it would do otherwise. The last-mentioned effect is responsible for the so-called stress-concentration ("Maltese cross") artefact seen in strain images of a hard inclusion in a soft background ( $\bullet$ Fig.5-7). It may also be the source of "edge-enhancement" effects ( $\bullet$ Fig. 8), which are generated also by the existence of slippery boundaries between structures (see next paragraph). Care is therefore needed to interpret the apparent positions of structures in the strain image, as they may be slightly displaced relative to the corresponding structures in the B-scan, as in 0 Fig. 8.

Care is also required when interpreting strain images for other reasons, including: the stiffness of out-of-plane structures that influence in-plane strain, a slippery boundary at a tissue discontinuity may show high axial strain at the boundary, as well as low and heterogeneous strain beyond the boundary [18-20], and fluid that can be displaced during a slow palpation produces a time-dependent reduction in the strain in nearby tissue [21].

Although there are many factors other than tissue elasticity that influence strain contrast, careful technique and image interpretation minimises their influence on judgement, and may even provide additional diagnostic information beyond that related to simple tissue elasticity; as with ultrasound imaging, a knowledgeable interpretation of artefacts can help extract more information from an image. Examples are artefacts mentioned above, namely stress concentrations which may increase the visibility of stiff structures ( $\bullet$ Fig. 5, 6, 8-10, and section on appearances of cysts on p.176), and the strain image characteristics at slippery boundaries ( Fig.9, 10). Another example is that the strain from a given force decreases with increasing force, i.e. the tissue gets harder the more force that is applied, because tissues are mechanically nonlinear (online appendix). It has been shown that the degree of nonlinearity is potentially a diagnostically useful tissue property [22-24]. Although stress measurement is re- 

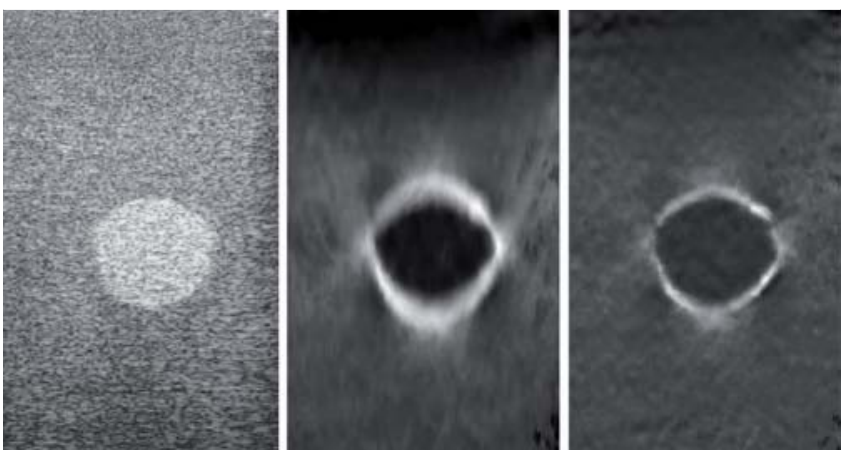

Fig. 9 An example of the trade-off between resolution and contrast discrimination, determined by the choice of window sizes both for displacementtracking and strain-estimation. Left: B-scan of a gelatine phantom containing a hyperechoic spherical inclusion. Middle: strain image with large displacement-tracking and strain-estimation windows, showing that the inclusion is stiffer (darker) than the background, that strain at the phantom's upper surface is reduced by friction with the probe, and that the inclusion has a slippery margin (indicated by the bright strain "halo" [20]). Right: strain image with small tracking and strain estimation windows, making it difficult to detect the strain contrast between inclusion and background, and the low strain near the upper surface of the phantom, but demonstrating resolution of the inclusion's slippery margin that is only slightly worse that that of the B-scan.

quired for a quantitative assessment of nonlinearity, it can be qualitatively judged during the periods of near constant rate of surface displacement with time ( $\bullet$ Fig. 6 ) by examining changes in strain-image lesion-to-background contrast, or graphs of mean inter-frame strain within a lesion, as a function of frame number in the absence of time averaging (i.e. with persistence switched off). Such graphs are plotted on-screen by at least one commercial SE system. Finally, time-dependent strain changes during a sustained compression may indicate a poroelastic or a viscoelastic response of the tissue (online appendix), which have been shown to provide excellent potential for characterisation of oedema [25] and as an additional biomechanical feature for distinguishing benign from malignant breast tumours [26], although current commercial SE systems are not optimised to observe these phenomena.

A confounding factor that is more difficult to account for is an intrinsic loss of shear modulus contrast when it is imaged as strain $[27,28]$. This is particularly true for soft regions buried in a stiff background, which are difficult to see in a strain image, a problem that has been termed the "egg shell" effect, where the stiff shell prevents the generation of strain inside the egg. In practice it will be difficult to know when this artefact is present, since it manifests for example as soft inclusions not appearing as soft as they really are. In the more complex but likely scenario of a soft background tissue containing a stiff tumour that has a very soft (e.g. necrotic) centre, so long as the centre is sufficiently echo rich to provide a strain image signal, the strain image is likely to underestimate the contrast for the soft centre and may even suggest that the centre is stiff relative to the background tissue. In the case of an echo-free tumour centre, shadow, or a cyst, other artefacts are generated that would obscure this effect. These are dealt with on p. 177 in the section on cysts.

\section{Strain image quality and factors that influence it}

The better the ultrasound image resolution the better the strain image resolution (though the latter is always slightly worse). It
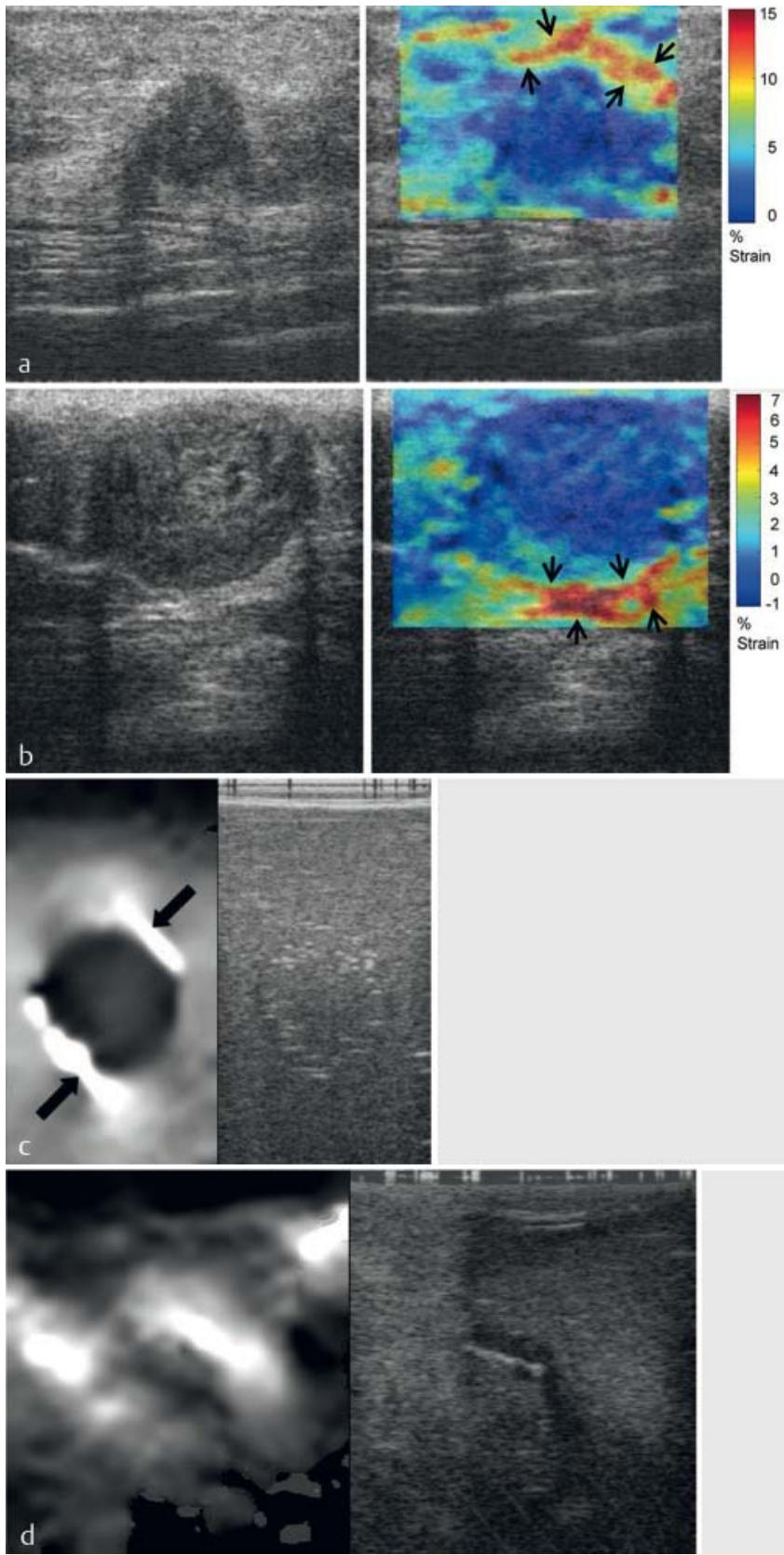

Fig. 10 a, b Ultrasound images (left) and strain elastograms (right) of two breast carcinomas where the tumour boundary is made more visible by likely stress concentration in surrounding tissue (black arrows) (from Brusseau et al. [66]). c, d Ultrasound images (right) and strain elastograms (left) of a gelatine phantom with a slippery inclusion $\mathbf{c}$ showing areas of high axial strain (arrows) at the slippery boundary, and an intraoperative scan of an intrinsic glioma $\mathbf{d}$ where areas of high axial strain were said to correspond to good cleavage planes upon surgical exploration (from Uff et al. [67]).

is determined by a trade-off with strain contrast discrimination, the trade-off being controlled by the choice of values for variables such as the window sizes for the displacement and strain estimators and the smoothing window, persistence, strain normalisation, and palpation speed and amplitude, some of which may not be under user control. A mechanical discontinuity, such as a slippery boundary at which displacement is discontinuous, can provide a good indication of resolution potential ( $\bullet$ Fig. 9 ). Image optimisation requires adjustment of many variables, but palpation speed and amplitude, and persistence, are particularly 

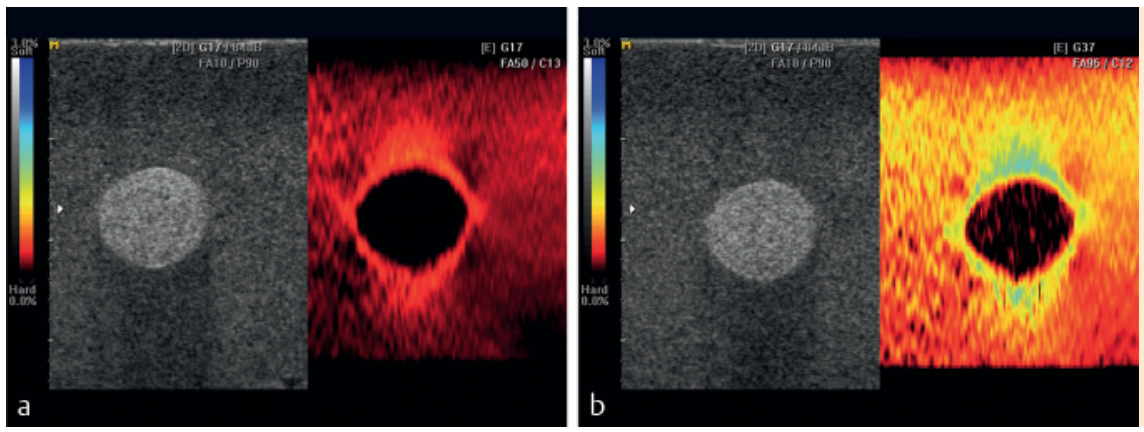

Fig. 11 Limited strain dynamic range requires palpation technique (speed and amplitude) to be adjusted so as to display the strain detail of interest. a Slow palpation is best for showing subtle details in the soft background material (e. g. stress concentrations and friction at the phantom surface) but fails to produce a strain signal that is visible above electronic noise in the stiff inclusion. b Fast palpation begins to show strain in the stiff inclusion but detail in the soft background is less apparent.

important. The reason for this, as demonstrated by Varghese and Ophir [29], is that only a limited range of inter-frame strains (in the region of $1-1.5 \%$ ) can be displayed with good SNR. It is difficult to predict the inter-frame strain at a given image location, since soft tissue will strain more than hard tissue. Although it is possible for a computer to adaptively select the inter-frame interval so as to maximise strain SNR on a pixel-by-pixel basis, it is not clear that commercial systems have yet implemented such a concept. Instead, accumulation over many frames (persistence) is commonly employed, which was shown by Doyley et al. [30] to allow excellent strain SNR to be maintained even at the high total strains induced at the point of maximum tissue displacement in freehand strain imaging. In practice therefore, to maximise strain image quality, palpation speed and amplitude should be adjusted interactively while viewing the image to achieve an inter-frame strain that best displays the desired tissue strain contrast, as explained in Fig. 11. Image reproducibility also varies with strain rate (and improves with use of persistence); Doyley et al. [30] found that freehand elastograms were most reproducible at low $\left(2.9 \% \mathrm{~s}^{-1}\right)$ strain rates (equivalent to about 0.5 compressions $\mathrm{s}^{-1}$ ) and, using a different system, Havre et al. [31] observed best reproducibility in the interval $1.3-2.0$ compressions $\mathrm{s}^{-1}$.

Filters are usually incorporated in quasi-static strain imaging systems to omit or replace poor quality frames or pixels. Examples of quality metrics that have been used for this purpose include the value of the correlation coefficient at the estimated displacement, the local spatial or temporal variation of displacement or strain, and the deviation of displacement values about the bestfit straight line within the strain estimation window. As the setting of the decision threshold for quality acceptance is arbitrary, this is under user control in many systems, and is often labelled as "gain" by analogy to colour Doppler gain or "rejection". Systems vary on how they deal with rejected pixels or frames which may, for example, be replaced with a mean or median of nearby pixels or frames. This type of processing is hidden from the user, but when combined with persistence it is a powerful way to improve image quality, and when enhanced with sophisticated strain normalisation methods it can produce extremely robust and reproducible freehand strain images of good quality regardless of whether the tissue displacement is uniaxial and in the ultrasound beam direction ( $\bullet$ Fig. 12), as would be the case when the transducer is held still and internal physiological motion utilised.

Many systems omit regions or frames of inadequate strain quality, with a varying approach to what is displayed instead. When the strain image is displayed separately from the echogram, inadequate quality regions may be replaced with a zero strain value (e. g. black) or with a colour mask that indicates that the data are of inadequate quality. Often the strain image is displayed as a col-

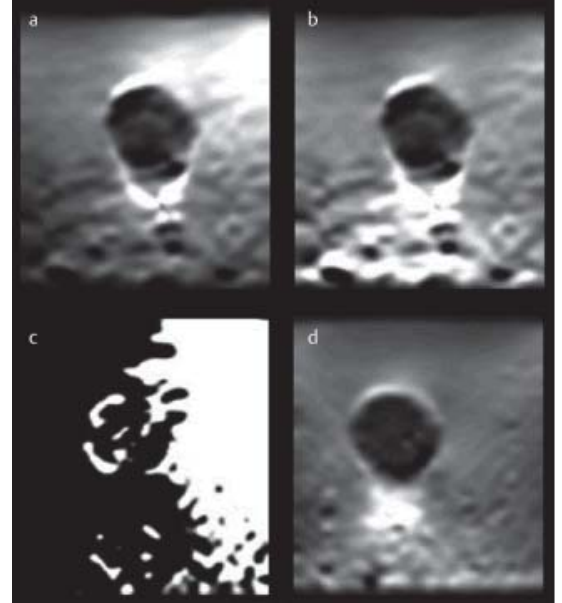

Fig. 12 The benefit of two-dimensional strain normalisation combined with adaptive frame averaging (persistence). a A phantom containing a stiff inclusion in a soft background imaged using strain calculated between two ultrasound frames acquired while palpating using a "best attempt" at moving the transducer with a smooth uniaxial motion. $\mathbf{b}$ The same image with two-dimensional strain normalisation applied. c As upper left but with a deliberately "poor technique" created by rocking the probe only (i. e. no axial motion). $\mathbf{d}$ Image obtained using the same technique as for bottom left but after applying two-dimensional strain normalisation combined with adaptive persistence, as described in Lindop et al. [68]. (Images courtesy of G. Treece).

our overlay, in which case it is common to display the ultrasound image in regions of inadequate strain quality. (Note that this is different to the use of a partially transparent colour elastogram overlaywhich has a different function, as discussed on p.182). Strain quality varies cyclically when using the probe to displace the tissue as shown in $\bullet$ Fig. 6 ; if the transducer speed is adjusted to produce a good quality image during the periods of steady motion, the quality will be poorest during the periods of minimum and maximum applied stress. In some systems the strain image may therefore flicker during palpation if persistence is low, turning off during these periods of insufficient inter-frame strain. In general, great care must be taken in setting the quality threshold as it determines the information seen in the strain image.

\section{Cysts and other echo-free regions}

SE artefacts may assist the discrimination of cystic from solid lesions by helping the observer perceive the absence of echoes. Without echoes, the tracking or Doppler algorithms detect random noise which produces strain noise, and when the sign of the strain is discarded by detecting its magnitude the strain noise appears as a high and rapidly fluctuating strain. The final appear- 


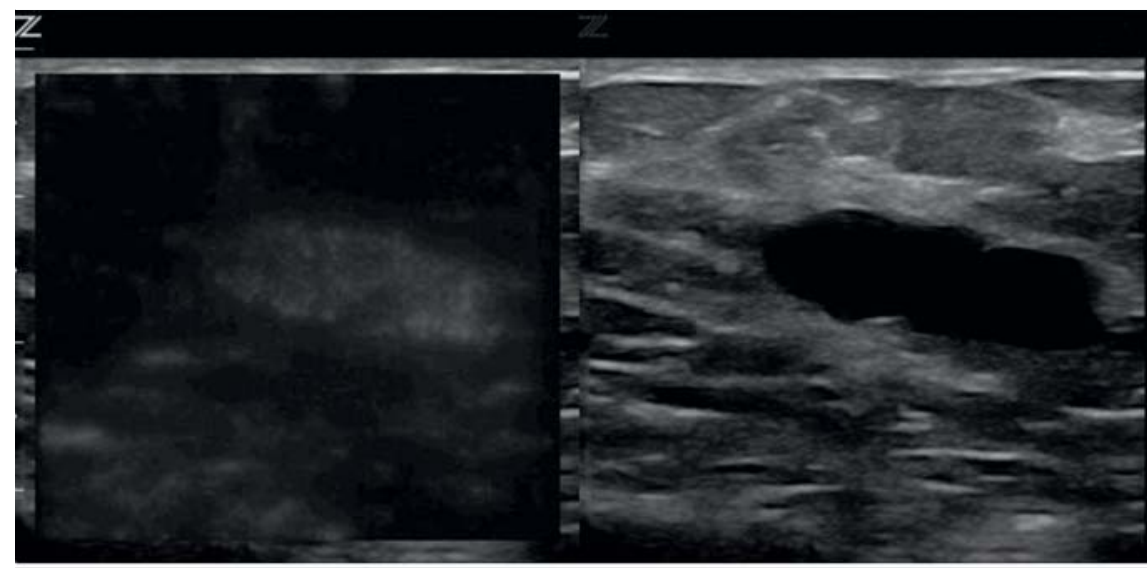

Fig. 13 Examples of cysts using one SE system. Strain images are on the left, conventional images on the right. a A large cyst imaged with low persistence (causing strain noise to fluctuate in real time), no palpation (causing very little tissue strain), small displacement tracking window size (resulting in a mottled strain noise pattern) and small strain estimator window with little smoothing (causing strain noise almost to fill the cyst). $\mathbf{b}$ A small cyst imaged with persistence, during palpation (causing the cyst boundary to show low strain and other tissues to show strain structure), and small window sizes for tracking and strain estimation. Strain noise is seen in the centre of the cyst which, in combination with the low strain boundary, generates the "target" or "bull's eye" appearance reported for

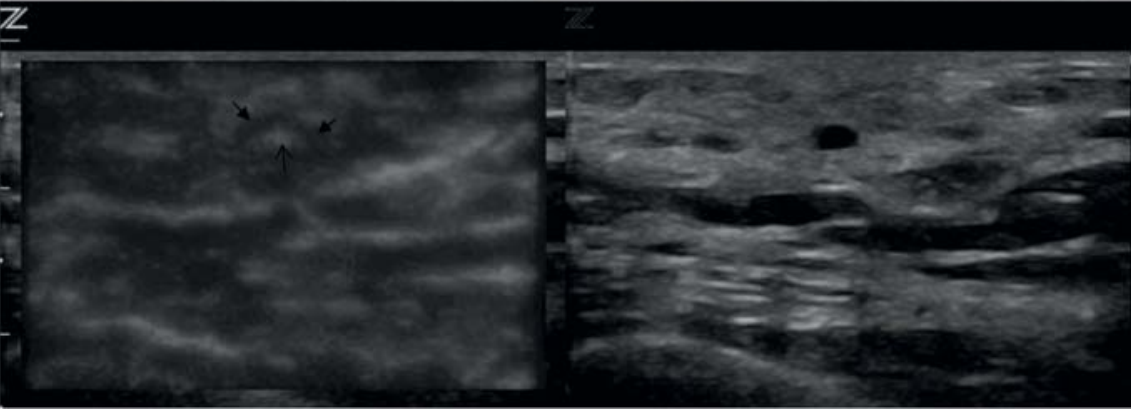
some systems. Recognition of this appearance may be assisted if there is high (real) anterior or posterior strain due to stress concentrations, which reinforce the deduction that despite the apparently high (artefactual) strain in the centre of the cyst, it is nevertheless a stiff structure.

ance of cysts depends strongly on an interaction between the size of the anechoic region and the combination of variables mentioned above in the section on strain image quality, some of which may have been chosen by the manufacturer. As a result, users of different scanners tend to describe different "characteristic" appearances of cysts. For example, if a cyst is stiffer than surrounding tissue it will appear dark on the SE image during palpation, possibly with anterior or posterior "bright-ups" due to stress concentration, and it may fill-in with the bright strain noise artefact. This may explain the origin of the "target" or "bull's-eye" appearance that has been described [32]. Depending on the system, it can be helpful to stop palpating and (if possible) switch strain persistence to zero and minimise quality rejection (or maximise "gain" if that is the label used for the rejection threshold). In the absence of endogenous tissue motion, this reduces the tissue strain to zero, leaving only rapidly fluctuating strain in the cyst. Alternatively, some systems allow the quality metric (e.g. correlation) to be viewed as an image. Again, this is best viewed in the absence of tissue strain. Some of these appearances are shown in $\bullet$ Fig. 13. Note that this "cyst detection" mode of strain imaging in part uses information already present in the ultrasound image of rapidly-fluctuating and spatially-fine random noise, which is best perceived in the ultrasound image by using high gain and zero persistence. It is currently not known whether strain imaging truly enhances ability to be sure that no echoes are present: it may simply be that observing the strain image is more convenient than adjusting the ultrasound image settings. Alternatively, strain imaging may be converting a difficult motion-assessment perceptual task to a simpler grey-scale or colour assessment, providing a benefit analogous to strain imaging's improvement over "visual relative motion assessment" of real-time ultrasonic images for elastic lesion detection [33].
Avoiding confounding factors in clinical quasi-static strain imaging

Conditions favourable to obtaining good quasi-static strain images representing tissue elasticity include:

- Close proximity of the target to the transducer $(<3-4 \mathrm{~cm})$

- Near homogeneous tissue (e.g. liver)

- No anatomical planes that allow slip movement anterior to or within the imaged region

- Some distance to tissue boundaries

- No structures present (e.g. large veins) that would damp the shear stress

- A broad stress source (in $2 \mathrm{D}$ ) relative to the width of the imaged region

- Knowing the position of the stress source relative to the imaged region

- A limited number of diagnostic targets

\section{Semi-quantitative analysis of strain images}

Methods for calculating elasticity based on strain observations represent an example of solving "the inverse problem" in elasticity imaging [34]. The simplest approach is to assume that stress is uniformly distributed and is everywhere equal to one, making the elastic modulus equal to the inverse of strain. Unfortunately, it is extremely difficult to palpate with the same force and, as described above, many different factors contribute to invalidating this assumption. This invalidates attempts to score or measure mean image brightness or colour within a lesion, and is important to keep in mind for the use of (a) scores of lesion-to-background strain contrast, (b) calculated strain ratios, (c) scores of the pattern of strain distribution and (d) calculated strain heterogeneity with a lesion, e.g. by strain histogram analysis. That is, the reference tissue selected for judgment of contrast or for calculating strain ratio should experience similar stress to the target $[31,35]$ and the judgement of strain pattern or heterogeneity 
should account for the expected stress distribution, both of which are difficult.

\section{Dynamic methods \\ $\nabla$}

Dynamic methods also use the tissue displacement estimation techniques employed in SE but differ in the method of applying the force or in the way that displacement is used ( $\bullet$ Table 1 ). The brief localised displacement induced by a focused acoustic radiation force impulse may be displayed directly. Alternatively, propagation of a transient displacement may be used to determine shear-wave speed. The dynamic nature of the force is essential in both cases.

\section{Acoustic radiation force impulse (ARFI) imaging: imaging tissue displacement induced by radiation force} Acoustic radiation force can create a localised displacement of a few microns in the ultrasound axial direction, which decays in a few ms [36]. Sufficient force for this purpose can be generated with a standard ultrasound scanner at depths of many centimetres by a sequence of rapid bursts of long (tens of $\mu \mathrm{s}$ ) focussed ultrasound pulses [37]. The displacement is measured at a known time after cessation of the push using RF-echo tracking, and displayed as a qualitative elastogram within a small box [38] ( $\triangle$ Fig. 14a). As the ultrasound beam creates the displacements, they are less user-dependent (i.e. more uniaxial) than those in hand-induced strain imaging. Compared to SE images, ARFI images have also been shown in phantoms to have better resolution, suffer less from stress concentration artefacts, possess better transfer of shear modulus contrast to image contrast, exhibit improved SNR at depth and be less influenced by the presence of slip movement anterior to the imaged region [39]. Some of these properties are illustrated in the images shown in 0 Fig. 14. Cysts appear very bright on ARFI images with a dark halo, i. e., similar to the appearance on SE when palpating ( $\bullet$ Fig. 13), although this is without the stress concentration "bright-ups" anterior and posterior to the cyst and it seems to be independent of whether the cyst contains internal echoes [40]. It seems that ARFI imaging does more than detect whether there is a lack of echoes (the radiation force may cause fluid to move) although displacement noise may well be displayed in the absence of echoes. Drawbacks to this technique include: although the displacement contrast is related to the elastic contrast, it depends also on absorption and reflection of the pushing beam (although artefacts from this can be useful, e.g. in highlighting calcifications); the contrast depends on the delay between the push and the displacement measurement; and transducer heating from the high power needed to create the pushing beam limits the frame rate with present technology. A commercial system, provided by Siemens, is based on this technique.

Transient elastography (TE): shear-wave speed measurement using a surface impulse

This method is known as transient elastography even though all of the dynamic methods use transient excitations. It employs a brief push (a small "thump") applied with an automated movement of the ultrasound transducer, which acts like a piston at the skin surface ( $\bullet$ Fig. 15). The strongest shear-wave arises from the edge of the piston: thus a disc-shaped piston approximates a ring source. A component of the wave from this ring converges on the ultrasound axis and after some distance travels

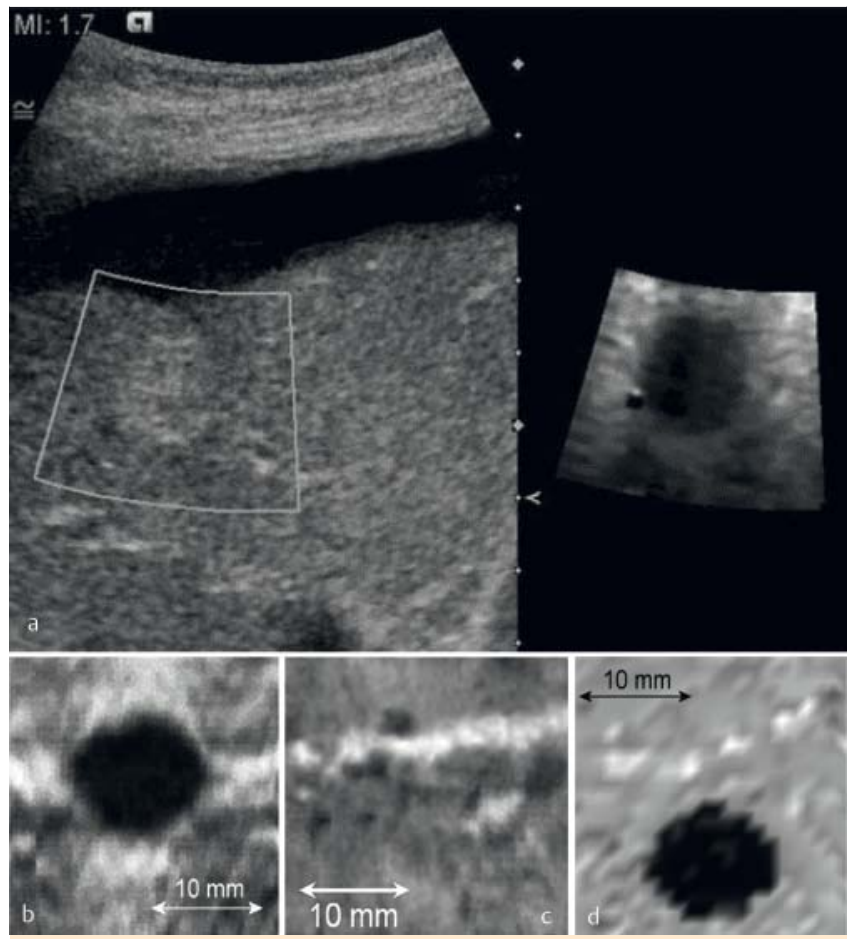

Fig. 14 a ARFI image (right) demonstrates a liver metastasis with higher contrast and clearer boundaries than on the B-mode image (left), despite the ascites. b Quasi-static SE of a gelatin phantom containing a stiff inclusion in a soft background, demonstrating high strain above and below and to the left and right of the inclusion, because of the stress concentration phenomenon described in the section on SE. c Quasi-static SE of same phantom after slicing the background gel anterior to the inclusion, demonstrating ability to detect the discontinuity (bright inclined band about one third from the top of the image) but not the stiff inclusion. $\mathbf{d}$ Same region of the phantom as for $\mathbf{c}$ but imaged using ARFI: showing good inclusion:background contrast, sharp inclusion boundaries, low background noise, and freedom from stress concentrations, despite the presence of the discontinuity. Note that the image in $\mathbf{d}$ was built-up slowly; each pixel required a separate ARFI push pulse with the pushing focus located at the pixel concerned (Image in a courtesy of Siemens. Images in b, $\mathbf{c}$ and $\mathbf{d}$ reproduced with permission from [39].).

down the axis at a speed close to the shear-wave speed. Its propagation may be followed on a single RF A-line at high pulse repetition frequency [41]. The shear displacement (in the ultrasound axial direction) versus depth may then be measured using $1 \mathrm{D}$ correlation tracking methods, and the speed of the wave obtained from a displacement "M-mode" ( $\odot$ Fig. 15) as the slope of a straight line (the oblique white line in the figure) that is automatically fitted to the approximately linear portion of the shear-wave arrival time versus depth. The definition of arrival time is arbitrary but the speed measurement will be accurate so long as the same reference point on the time-varying displacement is used for all depths and so long as the shape of the shear-wave does not change as it travels. The straight line avoids both the deep region where the displacement SNR is poor, due to the decay of the shear-wave amplitude with depth, and the superficial region where the apparent wave speed varies with depth (it is strongly influenced by the wave convergence angle which is determined by the size of the piston). A special algorithm rejects displacement $\mathrm{M}$-modes that are not linear enough, and the display allows the quality of the measurement to be judged. 


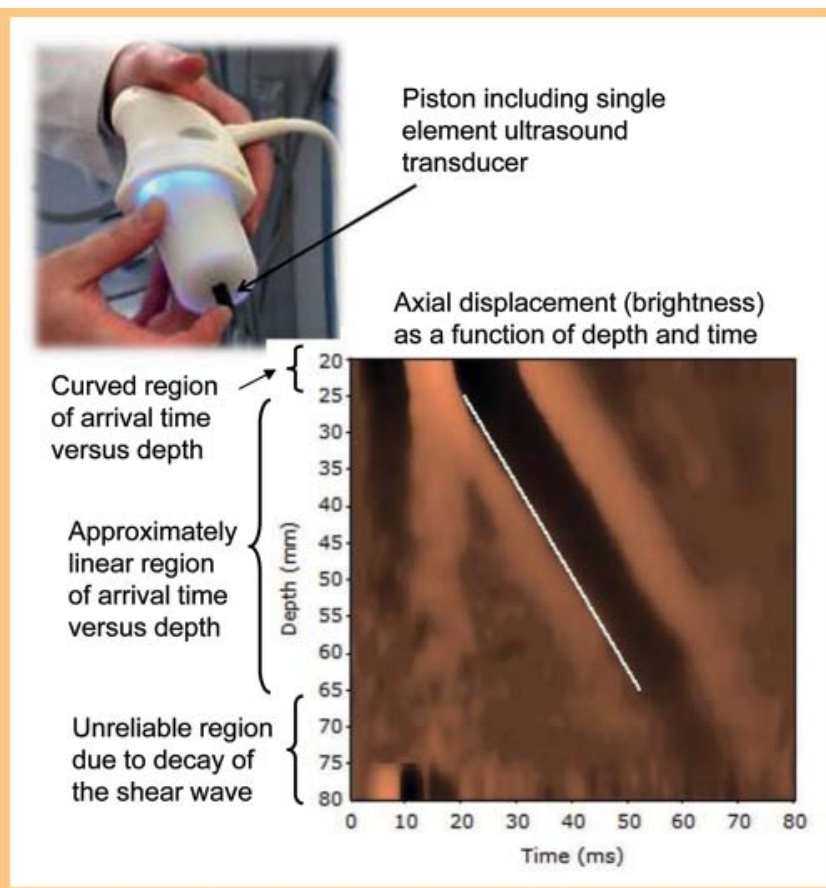

Fig. 15 Upper: The Echosens applicator contains a piston which includes the ultrasound transducer. Lower: a displacement "M-mode" image which shows axial displacement (brightness) as a function of depth and time. Shear-wave speed is measured as the slope of a straight line (in white) of best fit to the depth dependence of a suitable reference point (the "timeof-arrival") on the time-varying displacement.

The shear-wave speed can be converted to a Young's modulus value using the relation $E=3 \rho c_{s}^{2}$ (see online appendix), and the measurement given in $\mathrm{kPa}$ [41]. The system from Echosens is designed for easy use and measurement of liver tissue elasticity [42-45]. It provides a regional elasticity measurement (within the width of the ultrasound beam and the depth of shear-wave penetration of several $\mathrm{cm}$ ); it is not an ultrasound scanner and provides no conventional ultrasound anatomical images or elastograms. The system lacks $2 \mathrm{D}$ image guidance of the measurement, and it is not possible to propagate the shear-wave beyond fluid collections (ascites). It may be difficult to obtain sufficient signals in obese patients with the standard probe (3.5 MHz, $2 \mathrm{~mm}$ vibration amplitude) although the failure rate has been reduced by the introduction of a dedicated obese-patient probe (2.5 $\mathrm{MHz}, 3 \mathrm{~mm}$ vibration amplitude). On the other hand, the use of the ribs for support when generating the intercostal transient shear-wave limits the amount of prestress, which may be a confounding factor in elastography because tissue is elastically nonlinear, i. e. the more it is stressed the stiffer it gets. The transient surface displacement is also tightly controlled, as a single-cycle of a $50 \mathrm{~Hz}$ wave. This is expected to help reproducibility of the measurements because, as described in the online appendix, tissue elasticity and hence shear-wave speed depends on the frequency of vibration. Finally, since the shear-wave is not generated by acoustic radiation force, it is relatively easy to keep the ultrasound thermal and mechanical indices low.
Point shear-wave elastography (pSWE): shear-wave speed measurement at a location using acoustic radiation force

The type of localised transient displacement generated by ARFI, described on p.179, creates a transient shear-wave propagating with cylindrical symmetry away from the pushing-beam's axis and focus, being strongest at the depth of the pushing-beam's focus. The shear displacement ( $\bullet$ Fig. 3 ) is along the ultrasound imaging beam, allowing the use of correlation tracking or Doppler to measure the small displacements of the shear-wave and detect its time of arrival at lateral positions.

In the method of Nightingale et al. [46] the shear-wave is generated using ARFI, and its arrival time at nearby A-lines is measured to provide the average shear-wave speed between two points bounded by a measurement region of interest (ROI). The speed can be measured to depths of up to about $8 \mathrm{~cm}$ [47] and, as with $\mathrm{TE}$, is reported in units of $\mathrm{ms}^{-1}$ or converted to Young's modulus. Although ultrasound imaging is used to guide placement of the ROI, no elasticity images are produced. Nevertheless, the underlying concept is the basis for SWE described below. It is thus herein referred to as point SWE (pSWE), although it has been described in the literature as ARFI quantification. Commercial systems by Siemens and Philips are based on this technique.

An important application is in characterising diffuse liver disease where, unlike TE, there are no problems with propagating through ascites, and potentially fewer problems with obese patients and with patients with dysmorphic livers, given the guidance of conventional ultrasound.

\section{Shear-wave elastography: shear-wave speed imaging} (SWE, 2 D SWE or 3 D SWE) using acoustic radiation force SWE can be utilized to produce two- or three-dimensional quantitative images of shear-wave speed with a useful field of view (2 D SWE or $3 \mathrm{D}$ SWE). One such technique is implemented by SuperSonic Imagine. The acoustic radiation force focus is swept down the acoustic axis faster than the shear-wave speed, so as to generate tissue displacements (tens of $\mu \mathrm{m}$ ) at all positions along the acoustic axis almost simultaneously. This produces a shear-wave in the shape of a cone with a shallow angle (and hence is almost cylindrical), known as a Mach cone, that travels away from the push line, which spreads less and thus decays less rapidly with distance than that from a single pushing focus. An ultrafast scanner achieves an ultrasound frame rate of up to $20 \mathrm{kHz}$ by transmitting a plane wave and focusing only on receive, so that each ultrasound echo image is created with a single transmit pulse. This high frame rate allows the shear-waves to be followed in real time in $2 \mathrm{D}$, and RF-echo tracking over a grid of points produces a displacement movie ( $\bullet$ Fig. 16) from which a small map of shear-wave time-of-arrival can be created. Its size depends on displacement SNR and the attenuation of the shearwave. It is then analysed by detecting the differences between arrival times at different lateral positions to create a part of the final image of shear-wave speed. The process is repeated for a number of different push lines to create a final quantitative elasticity image in a box, which is presented as a colour overlay on the Bmode image in units of $\mathrm{ms}^{-1}$, or converted to Young's modulus in $\mathrm{kPa}$ as for TE $[48,49]$. The maximum size of the elastogram box is approximately $2-3 \mathrm{~cm}$ of side length with a linear transducer, and $9 \times 4 \mathrm{~cm}$ with a convex transducer. Thus the system uses three frame rates: the standard B-mode echo image, a hidden ultrafast echo image used to track the displacement, and the shearwave elastogram. Elastograms are created at $3-4 \mathrm{~Hz}$ although the 


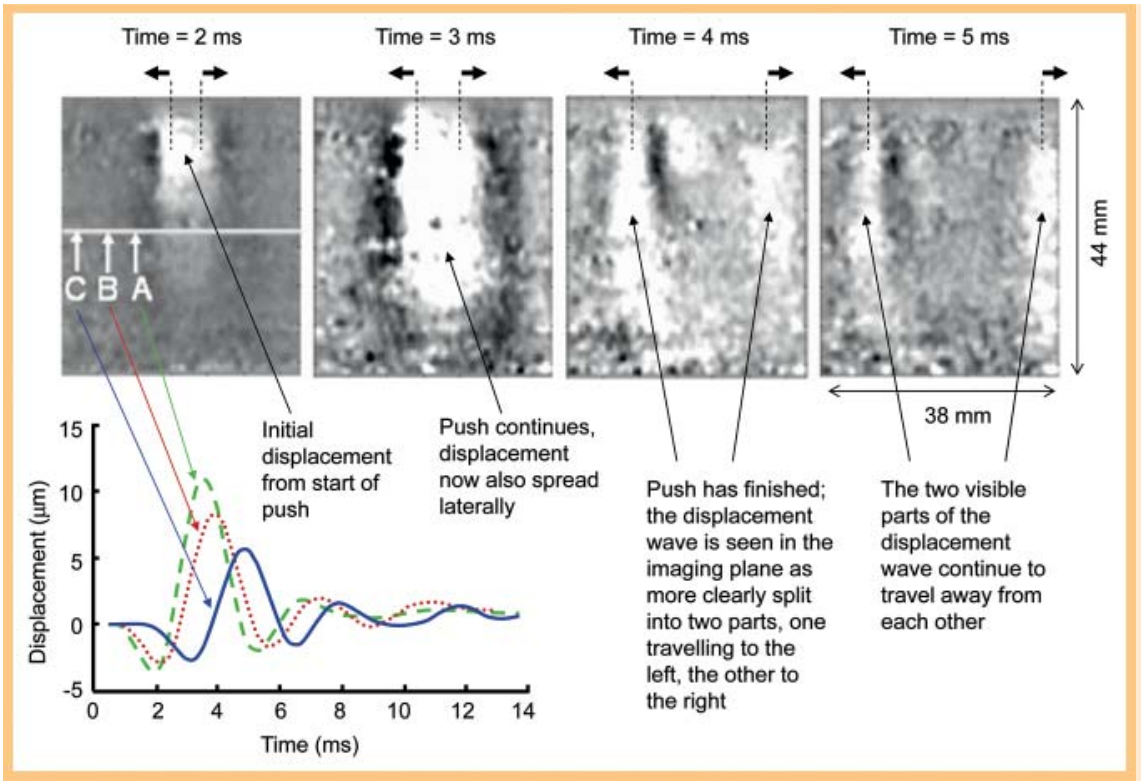

Fig. 16 Top row: four example frames (at 2, 3, 4 and $5 \mathrm{~ms}$ after the start of the radiation force push) from an axial displacement movie showing a conical shear wave developing and then propagating in the breast in vivo. The graph lower left shows the displacement-versus-time waveforms observed as the shear wave passes the three points, A, B and C, in the upper-left image. The difference in the times of arrival of the shear wave at each of these different locations allows the average shear-wave speed to be determined for each of the paths between each of these locations. (Adapted from Tanter et. al. [50], courtesy of Elsevier).

persistence needed for noise reduction reduces the SWE frame rate. Spatial resolution is a little worse than in strain imaging and ARFI displacement imaging but the elastograms are quantitative and display no stress concentration artefacts ( $\bullet$ Fig. 17). Penetration in the breast [50] is about $3.5 \mathrm{~cm}$ with a linear transducer, and in the liver about $8 \mathrm{~cm}$ with a convex transducer [51, 52]. A quality-factor masking of SWE noise allows the B-mode image to show through, the threshold for which is under user control labelled as SWE gain. The potential for measuring the viscous component of the shear modulus of tissue elements, by measuring the frequency dependence of shear-wave speed, has also been demonstrated $[50,53]$.

The Siemens Acuson S3000 also provides a two-dimensional SWE image. The image is acquired in under $700 \mathrm{~ms}$ by translating the pushing focus to cover up to 256 acquisition lines $(38 \mathrm{~mm}$ elastogram-box width) where, for each, pSWE is carried out at a number of positions around the pushing focus. A static quantitative SWE image is therefore displayed. As with ARFI imaging, at present, after acquiring an image, time is need to allow the transducer to cool before another image may be generated.

Operator dependence of SWE is small, although care is needed to minimise probe pressure when imaging superficial tissues so as to avoid a high shear-wave speed artefact near the surface due to localised pre-stress which causes the superficial tissue to stiffen because of the nonlinear stress-strain character of tissue. For SWE of the liver, as with TE, intercostal imaging may help prevent pre-stress as pressure on the ribs is not easily transmitted to the liver. Factors affecting data quality and producing speed errors or loss of signal, include: strength of the push, variations in attenuation, absorption and reflection of the pushing beam, ultrasound scatterer density, tissue continuity, very high or very low shearwave speed, and shear-wave scattering, reflection or refraction. Thus, although shear-waves do not propagate through clear non-viscous liquids, care is needed in interpreting lack of SWE signal as indicating a cyst. Partial volume effects also need to be considered when interpreting the images. Finally, shear-wave propagation near boundaries and within thin layers may invalidate the assumed relationship between their speed and elastic modulus. More detail on these phenomena may be found in the online appendix of this paper. A full characterisation of the artefacts associated with SWE has yet to be conducted.

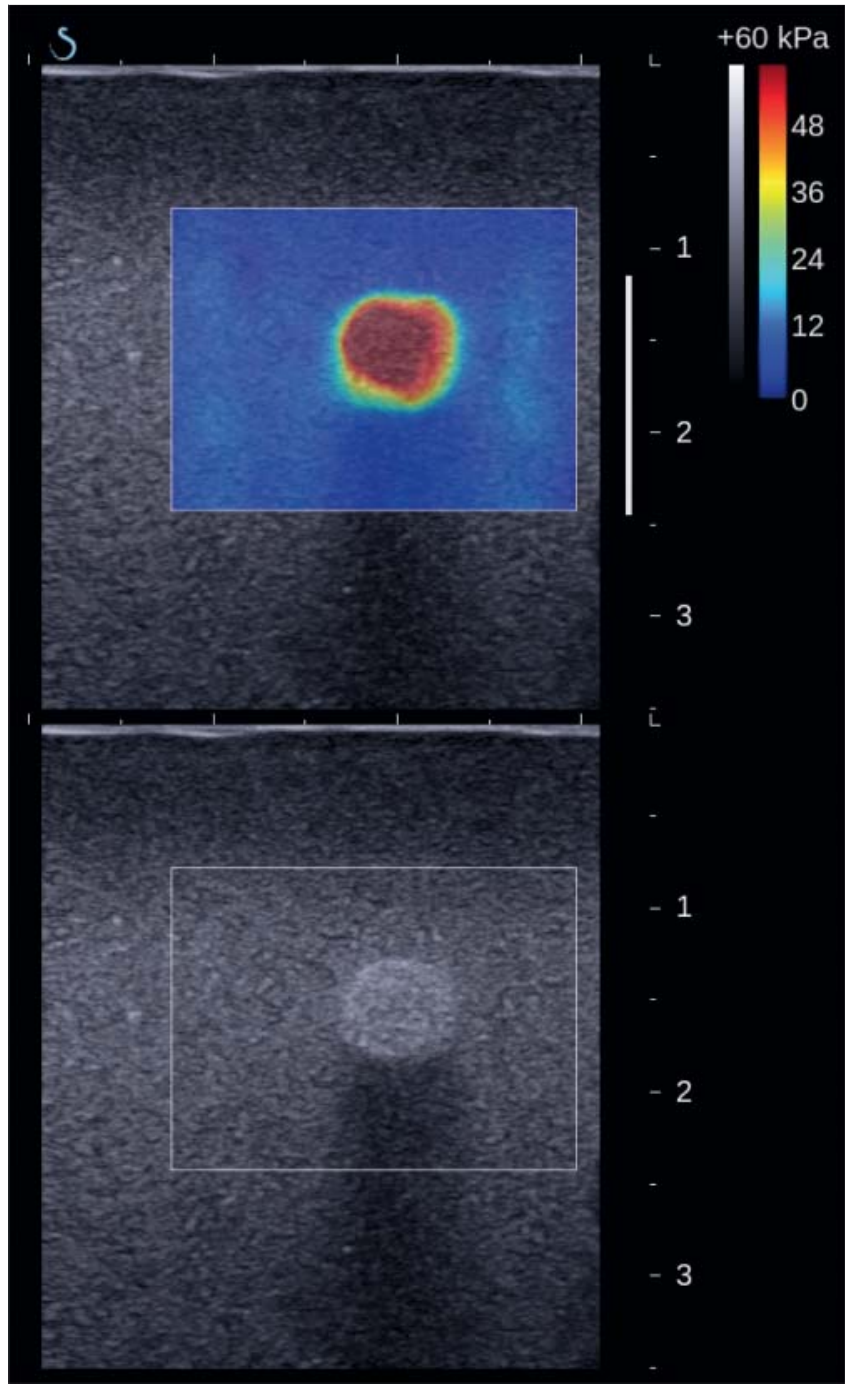

Fig.17 Example of SWE of same phantom as imaged in Fig. 9, 11. Lower: a B-scan showing a hyperechoic and attenuating inclusion (axial distance scale in centimetres). Upper: overlay of a transparent coloured SWE on the B-scan, showing that the inclusion is homogeneously hard within a background that is homogeneously soft (colour scale shows Young's modulus in $\mathrm{kPa}$ ). Note: the images have been cropped from the originals. 


\section{Recommendations for display}

A semi-transparent overlay of the elastogram onto the B-mode echogram assists judgement of the spatial relationships of ultrasound imaging and elastographic information. Judgements of elastographic detail, contrast or stiffness are best made using a side-by-side display or by setting the elastogram to be opaque, to avoid confusion between elastographic and ultrasound imaging detail and brightness.

Selection of grey-scale display for the elastogram, or a colour scale with a monotonically increasing intensity, avoids artefactual contouring structure in the image and is best for judging elastographic detail. A colour scale in which hue varies helps judgement of hardness or contrast; a commonly used scale of this type runs from red to blue via intervening colours such as yellow and green. Currently, however, there are differences between manufacturers, and greater standardisation is needed as to whether red or blue means soft and whether the top of the scale bar indicates soft or hard. Clearer indication, with larger fonts where necessary, of hard and soft on the scale would also be helpful. Finally, care is needed when interpreting displays that code the elastogram as black where quality is poor since this may cause poor quality to be confused with a low value of the quantity imaged if a colour scale is used in which black represents the bottom of the scale.

\section{Safety considerations}

Quasi-static strain imaging and shear-wave methods based on force applied by dynamic surface displacement have identical ultrasound safety considerations to conventional ultrasound imaging modes. Radiation force based elastography operates with higher TI but is measured to be within AIUM limits and safety considerations are similar to Doppler mode, i. e. normal due diligence should be employed concerning sensitive tissues such as the eye and fetus, and when bone may be present in the pushing beam [54-56].

\section{Conclusion}

Effective use of elastography requires knowledge of the underlying physics and technology, which is made difficult by the complexity of the subject and the variety of technologies available. This complexity, however, brings opportunities, which the research community is pursuing with enthusiasm. As a result, the technology for elastography is likely to undergo considerable development beyond the current techniques available. Substantial improvements can be expected in image quality, ease of use, quantification and range of tissue characteristics that are measurable. For further reading, reviews include Doyley et al. [33], Palmeri and Nightingale [38], Wells and Liang [57], Parker et al. [58], Bamber [59], Bamber et al. [60], Gao et al. [61], Greenleaf et al. [62] and Ophir et al. [27, 63, 64].

\section{List of abbreviations}

\section{$\nabla$}

$2 \mathrm{D}$ two-dimensions

$3 \mathrm{D}$ three-dimensions

ARFI acoustic radiation force impulse

EFSUMB European Federation of Societies for Ultrasound in Medicine and Biology

kPa kilopascal

pSWE point SWE

$\mathrm{RF}$ radio frequency

ROI region of interest

SE strain elastography

SNR signal-to-noise ratio

SR strain ratio

SRI strain-rate imaging

SWE shear-wave elastography

TE transient elastography

\section{Affiliations}

1 The Joint Department of Physics, Division of Radiotherapy and Imaging, The Institute of Cancer Research and The Royal Marsden NHS Foundation Trust, Sutton, UK

2 Div. of Radiology, Imperial and Kings Colleges, London, UK

3 Div. Gastroenterology \& Oncology, Caritas Krankenhaus, Bad Mergentheim, Germany

${ }^{4}$ Department of Internal Medicine I, Endocrinology and Diabetology, GoetheUniversity, Frankfurt, Germany

${ }^{5}$ Div. of Radiology, University of Pavia, Policlinico San Matteo, Italy

${ }^{6}$ Department of Radiological Sciences, Oncology and Pathology, Policlinico Umberto I, Univ. Sapienza, Rome, Italy

Adult Radiology, Paris Descartes University \& Necker University Hospital, France, Institut Langevin - Ondes et Images ESPCI Paris Tech, CNRS UMR 7587 INSERM U 979

8 Department of Radiology, GB Rossi University Hospital, University of Verona, Italy

9 Radiologist, University Hospital Heraklion, Crete, Greece

${ }^{10}$ Ecole Supérieure de Physique et de Chimie de la Ville de Paris, France

11 Department of Internal Medicine I, Gastroenterology and Hepatology, Goethe-University, Frankfurt, Germany

12 National Centre for Ultrasound in Gastroenterology, Department of Medicine, Haukeland University Hospital, Bergen, Norway and Department of Clinical Medicine, University of Bergen, Norway

${ }^{13}$ National Centre for Ultrasound in Gastroenterology, Department of Medicine, Haukeland University Hospital, Bergen, Norway

14 Department of Internal Medicine, Krankenhaus Märkisch Oderland, Strassberg, Germany

15 Department of Radiology, Innsbruck Medical University, Austria

${ }^{16}$ Department of Obstetrics and Gynecology, Breast unit, University Greifswald, Germany

17 Department of Gastroenterology, Research Center of Gastroenterology and Hepatology, University of Medicine and Pharmacy Craiova, Romania

18 Unit of Breast Imaging and Interventions, University Hospital SchleswigHolstein Campus Kiel, Germany

19 Department of Gastroenterology and Hepatology, University of Medicine and Pharmacy "Victor Babes" Timisoara, Romania

20 Div. Internal Medicine, University of Bologna, Bologna Italy

\section{Acknowledgements \\ $\nabla$}

The unrestricted financial support provided by the following companies (listed in alphabetical order) to support the preparation of this document is gratefully acknowledged:

BK Medical, Echosens, Esaote, GE Healthcare, Hitachi Aloka, Philips, Siemens, SuperSonic Imagine and Toshiba

Links to the industrial webpages describing in greater details proprietary elastographic technologies can be accessed through the the EFSUMB web portal (www.efsumb.org) section on guidelines (elastography) at this link: http://www.efsumb.org/guidelines/ elasto-manufacturers-links.pdf 


\section{References}

1 Wilkins RH. Neurosurgical Classic. Xvii. J Neurosurg 1964; 21: $240-$ 244

2 Claudon M, Dietrich CF, Choi BI et al. Guidelines and Good Clinical Practice Recommendations for Contrast Enhanced Ultrasound (CEUS) in the Liver - Update 2012. Ultraschall in Med 2013; 34: 11 - 29

3 Piscaglia F, Nolsoe C, Dietrich CF et al. The EFSUMB Guidelines and Recommendations on the Clinical Practice of Contrast Enhanced Ultrasound (CEUS): update 2011 on non-hepatic applications. Ultraschall in Med 2012; 33: 33 - 59

4 Cosgrove D, Piscaglia F, Dietrich CF et al. EFSUMB Guidelines and Recommendations on the Clinical Use of Elastography. Part 2: Clinical Applications. Ultraschall in Med 2013, in press

5 Hoskins PR. In: Hoskins PR, Thrush A, Martin K et al. (eds) Diagnostic ultrasound physics and equipment London: Greenwich Medical Media; 2003

6 Hill CR. In: Hill CR, Bamber JC, ter HaarGR (eds) Physical Principles of Medical Ultrasonics 2nd ed. Chichester: John Wiley; 2004

7 Szabo TL. Diagnostic ultrasound imaging: inside out. Burlington: Elsevier Academic Press; 2004

8 Shung KK. Diagnostic ultrasound: imaging and blood flow measurement. Boca Raton: CRC Press; 2006

9 Cobbold RSC. Foundations of biochemical ultrasound. Oxford: Oxford University Press; 2007

10 Bamber JC, Miller N, Tristam M. Diagnostic ultrasound. In: Flower MA (ed) Webb's Physics of Medical Imaging Bristol: Taylor \& Francis; 2012

11 Odegaard S, Gilja OH, Gregersen H. Basic and new aspects of gastrointestinal ultrasonography. In: Odegaard S, Gilja OH, Gregersen $\mathrm{H}$ (eds) Singapore: Wold Scientific Publisher; 2005

12 Sarvazyan A, Hill CR. Physical chemistry of the ultrasound-tissue intereaction. In: Hill CR, Bamber JC, ter HaarGR (eds) Physical Principles of Medical Ultrasonics 2nd ed. Chichester: John Wiley; 2004, 223-235

13 Eckersley RJ, Bamber JC. Methodology for imaging time-dependent phenomena. In: Hill CR, Bumber JC, ter Haar GR (eds) Physical Principles of Medical Ultrasonics 2nd ed. Chichester: John Wiley; 2004, $304-335$

14 Housden RJ, Chen L, Gee AH et al. A new method for the acquisition of ultrasonic strain image volumes. Ultrasound Med Biol 2011; 37: 434 441

15 Sutherland GR, Stewart MJ, Groundstroem KW et al. Color Doppler myocardial imaging: a new technique for the assessment of myocardia function. J Am Soc Echocardiogr 1994; 7: 441 - 458

16 Gilja $\mathrm{OH}$, Heimdal A, Hausken T et al. Strain during gastric contractions can be measured using Doppler ultrasonography. Ultrasound Med Biol 2002; $28: 1457-1465$

17 Ahmed AB, Matre K, Hausken $T$ et al. Rome III subgroups of functional dyspepsia exhibit different characteristics of antral contractions measured by strain rate imaging - a pilot study. Ultraschall in Med 2012; 33: E233-E240

18 Thitaikumar A, Ophir J. Effect of lesion boundary conditions on axial strain elastograms: a parametric study. Ultrasound Med Biol 2007; 33: $1463-1467$

19 Chakraborty A, Bamber JC, Dorward NL. Slip elastography: a novel method for visualising and characterizing adherence between two surfaces in contact. Ultrasonics 2012; 52: 364-376

20 Garcia L, Fromageau J, Bamber J et al. Further characterisation of changes in axial strain elastograms due to the presence of slippery tumor boundaries part 1: simulation study. In: Proceedings of the Ninth International Conference on the Ultrasonic Measurement and Imaging of Tissue Elasticity. Snowbird, Utah, USA: www.elasticityconference. org/prior_conf/2010/2010conf.htm 2010; 114

21 Garcia L, Fromageau J, Bamber J et al. Further characterisation of changes in axial strain elastograms due to the presence of slippery tumor boundaries part 2: experimental verification. In: Proceedings of the Ninth International Conference on the Ultrasonic Measurement and Imaging of Tissue Elasticity. Snowbird, Utah, USA: International Tissue Elasticity Conference; www.elasticityconference.org/prior_conf/ 2010/2010conf.htm 2010; 115

22 Goenezen S, Dord JF, Sink Z et al. Linear and nonlinear elastic modulus imaging: an application to breast cancer diagnosis. IEEE Trans Med Imaging 2012; 31: $1628-1637$

23 Krouskop TA, Wheeler TM, Kallel $F$ et al. Elastic moduli of breast and prostate tissues under compression. Ultrason Imaging 1998; 20 : $260-274$
24 Oberai AA, Gokhale NH, Goenezen S et al. Linear and nonlinear elasticity imaging of soft tissue in vivo: demonstration of feasibility. Phys Med Biol 2009; 54: 1191 - 1207

25 Berry GP, Bamber JC, Mortimer PS et al. The spatio-temporal strain response of oedematous and nonoedematous tissue to sustained compression in vivo. Ultrasound Med Biol 2008; 34: 617-629

26 Oiu Y, Sridhar M, Tsou JK et al. Ultrasonic viscoelasticity imaging of nonpalpable breast tumors: preliminary results. Acad Radiol 2008; 15: $1526-1533$

27 Ophir J, Alam SK, Garra BS et al. Elastography: imaging the elastic properties of soft tissues with ultrasound. J Med Ultrasonics 2003; 29 : $155-171$

28 Ponnekanti H, Ophir J, Huang Y et al. Fundamental mechanical limitations on the visualization of elasticity contrast in elastography. Ultrasound Med Biol 1995; 21: 533-543

29 Varghese T, Ophir J. A theoretical framework for performance characterization of elastography: the strain filter. IEEE Trans Ultrason Ferroelectr Freq Control 1997; 44: $164-172$

30 Doyley MM, Bamber JC, Fuechsel F et al. A freehand elastographic imaging approach for clinical breast imaging: system development and performance evaluation. Ultrasound Med Biol 2001; 27: 1347-1357

31 Havre RF, Elde E, Gilja $\mathrm{OH}$ et al. Freehand real-time elastography: impact of scanning parameters on image quality and in vitro intra- and interobserver validations. Ultrasound Med Biol 2008; 34: 1638-1650

32 Barr RG, Lackey AE. The utility of the "bull's-eye" artifact on breast elasticity imaging in reducing breast lesion biopsy rate. Ultrasound Q 2011; 27: $151-155$

33 Miller NR, Bamber JC. Thresholds for visual detection of Young's modulus contrast in simulated ultrasound image movies. Phys Med Biol 2000; 45: 2057-2079

34 Doyley MM. Model-based elastography: a survey of approaches to the inverse elasticity problem. Phys Med Biol 2012; 57: R35-R73

35 Havre RF, Waage JR, Gilja $\mathrm{OH}$ et al. Real-time elastography: strain ratio measurements are influenced by the position of the reference area. Ultraschall in Med 2012; 33: 559-568

36 Sarvazyan AP, Rudenko OV, Swanson SD et al. Shear wave elasticity imaging: a new ultrasonic technology of medical diagnostics. Ultrasound Med Biol 1998; 24: 1419-1435

37 Nightingale K, Soo MS, Nightingale $R$ et al. Acoustic radiation force impulse imaging: in vivo demonstration of clinical feasibility. Ultrasound Med Biol 2002; 28: 227-235

38 Palmeri ML, Nightingale KR. Acoustic radiation force-based elasticity imaging methods. Interface Focus 2011; 1: 553-564

39 Melodelima D, Bamber JC, Duck FA et al. Transient elastography using impulsive ultrasound radiation force: a preliminary comparison with surface palpation elastography. Ultrasound Med Biol 2007; 33: 959969

40 Meng $W$, Zhang $G$, Wu $C$ et al. Preliminary results of acoustic radiation force impulse (ARFI) ultrasound imaging of breast lesions. Ultrasound Med Biol 2011; 37: 1436-1443

41 Sandrin L, Tanter M, Gennisson JL et al. Shear elasticity probe for soft tissues with 1-D transient elastography. IEEE Trans Ultrason Ferroelectr Freq Control 2002; 49: 436 - 446

42 Castera L, Vergniol J, Foucher J et al. Prospective comparison of transient elastography, Fibrotest, APRI, and liver biopsy for the assessment of fibrosis in chronic hepatitis C. Gastroenterology 2005; 128: $343-350$

43 Foucher J, Chanteloup E, Vergniol J et al. Diagnosis of cirrhosis by transient elastography (FibroScan): a prospective study. Gut 2006; 55: 403 408

44 Fraquelli M, Rigamonti C, Casazza G et al. Reproducibility of transient elastography in the evaluation of liver fibrosis in patients with chronic liver disease. Gut 2007; 56: 968 -973

45 Rockey DC. Noninvasive assessment of liver fibrosis and portal hypertension with transient elastography. Gastroenterology 2008; 134: 8 14

46 Nightingale K, Palmeri M, Trahey G. Analysis of contrast in images generated with transient acoustic radiation force. Ultrasound Med Biol 2006; 32: $61-72$

47 Palmeri ML, Wang MH, Rouze NC et al. Noninvasive evaluation of hepatic fibrosis using acoustic radiation force-based shear stiffness in patients with nonalcoholic fatty liver disease. J Hepatol 2011; 55: 666 672

48 Bercoff J, Pernot $M$, Tanter $M$ et al. Monitoring thermally-induced lesions with supersonic shear imaging. Ultrason Imaging 2004; 26 : $71-84$ 
49 Bercoff J, Tanter M, Fink M. Supersonic shear imaging: a new technique for soft tissue elasticity mapping. IEEE Trans Ultrason Ferroelectr Freq Control 2004; 51: 396-409

50 Tanter M, Bercoff J, Athanasiou A et al. Quantitative assessment of breast lesion viscoelasticity: initial clinical results using supersonic shear imaging. Ultrasound Med Biol 2008; 34: 1373-1386

51 Muller M, Gennisson JL, Deffieux T et al. Quantitative viscoelasticity mapping of human liver using supersonic shear imaging: preliminary in vivo feasibility study. Ultrasound Med Biol 2009; 35: 219-229

52 Bavu E, Gennisson JL, Couade $M$ et al. Noninvasive in vivo liver fibrosis evaluation using supersonic shear imaging: a clinical study on 113 hepatitis C virus patients. Ultrasound Med Biol 2011; 37: 1361 - 1373

53 Deffieux T, Montaldo G, Tanter $M$ et al. Shear wave spectroscopy for in vivo quantification of human soft tissues visco-elasticity. IEEE Trans Med Imaging 2009; 28: $313-322$

54 Palmeri ML, Nightingale KR. On the thermal effects associated with radiation force imaging of soft tissue. IEEE Trans Ultrason Ferroelectr Freq Control 2004; 51: 551-565

55 Skurczynski MJ, Duck FA, Shipley JA et al. Evaluation of experimental methods for assessing safety for ultrasound radiation force elastography. Br J Radiol 2009; 82: 666-674

56 Tabaru M, Yoshikawa H, Azuma T et al. Experimental study on temperature rise of acoustic radiation force elastography. J Med Ultrasonics 2012; 39: 137-146

57 Wells PN, Liang HD. Medical ultrasound: imaging of soft tissue strain and elasticity. J R Soc Interface 2011; 8: 1521 - 1549

58 Parker KJ, Doyley MM, Rubens DJ. Imaging the elastic properties of tissue: the 20 year perspective. Phys Med Biol 2011; 56: R1 - R29
59 Bamber JC. Ultrasound elasticity imaging: definition and technology. Eur Radiol 1999; 9: S327-S330

60 Bamber JC, Barbone PE, Bush NL et al. Progress in freehand elastography of the breast. IEICE Trans Inf Syst 2002; 85: 5-14

61 Gao L, Parker KJ, Lerner RM et al. Imaging of the elastic properties of tissue - a review. Ultrasound Med Biol 1996; 22: 959-977

62 Greenleaf JF, Fatemi M, Insana M. Selected methods for imaging elastic properties of biological tissues. Annu Rev Biomed Eng 2003; 5: 57 - 78

63 Ophir J, Alam SK, Garra B et al. Elastography: ultrasonic estimation and imaging of the elastic properties of tissues. Proc Inst Mech Eng H 1999; 213: $203-233$

64 Ophir J, Garra B, Kallel F et al. Elastographic imaging. Ultrasound Med Biol 2000; 26: S23-S29

65 Kolen AF. Elasticity imaging for monitoring thermal ablation therapy in liver. In: PhD Thesis: University of London; 2003

66 Brusseau E, Detti V, Coulon A et al. A two-dimensional locally regularized strain estimation technique: preliminary clinical results for the assessment of benign and malignant breast lesions. In: D'hooge J, Doyley M (eds) Medical Imaging 2011: Ultrasonic Imaging, Tomography, and Therapy Lake buena vista, Florida: SPIE Proceedings; 2011, 79680J

67 Uff C, Garcia L, Fromageau J et al. Real-time ultrasound elastography in neurosurgery. In: Yuhas MP (ed) 2009 IEEE International Ultrasonics Symposium Rome: 2009, 467-470

68 Lindop JE, Treece GM, Gee AH et al. An intelligent interface for freehand strain imaging. Ultrasound Med Biol 2008; 34: 1117-1128 